REVISTA DE LA

UNIÓN MATEMÁTICA ARGENTINA

Vol. 59, No. 2, 2018, Pages 443-469

Published online: August 9, 2018

\title{
ON FAMILIES OF HOPF ALGEBRAS WITHOUT THE DUAL CHEVALLEY PROPERTY
}

\author{
NAIHONG HU AND RONGCHUAN XIONG
}

\begin{abstract}
Let $\mathrm{k}$ be an algebraically closed field of characteristic zero. We construct several families of finite-dimensional Hopf algebras over $\mathbb{k}$ without the dual Chevalley property via the generalized lifting method. In particular, we obtain 14 families of new Hopf algebras of dimension 128 with non-pointed duals which cover the eight families obtained in our unpublished version, arXiv:1701.01991 [math.QA].
\end{abstract}

\section{INTRODUCTION}

Let $\mathbb{k}$ be an algebraically closed field of characteristic zero. This work is a contribution to the classification of finite-dimensional Hopf algebras over $\mathbb{k}$ without the dual Chevalley property, that is, the coradical is not a subalgebra. Until now, there are few classification results on such Hopf algebras without pointed duals, with some exceptions in [15]. More examples are needed to get a better understanding of the structures of such Hopf algebras.

Our strategy follows the principle proposed by Andruskiewitsch and Cuadra [3], that is, the so-called generalized lifting method as a generalization of the lifting method introduced by Andruskiewitsch and Schneider in [7. Let $A$ be a Hopf algebra over $\mathrm{k}$ without the dual Chevalley property. Andruskiewitsch and Cuadra 3 . replaced the coradical filtration $\left\{A_{(n)}\right\}_{n \geq 0}$ with the standard filtration $\left\{A_{[n]}\right\}_{n \geq 0}$, which is defined recursively by $A_{[n]}=A_{[n-1]} \wedge A_{[0]}$, where $A_{[0]}$ is the subalgebra generated by the coradical $A_{0}$. Under the assumption that $S_{A}\left(A_{[0]}\right) \subseteq A_{[0]}$, it turns out that the standard filtration is a Hopf algebra filtration, and the associated graded coalgebra gr $A=\oplus_{n=0}^{\infty} A_{[n]} / A_{[n-1]}$ with $A_{[-1]}=0$ is a Hopf algebra. Denote by $\pi: \operatorname{gr} A \rightarrow A_{[0]}$ the canonical projection which splits the inclusion $i: A_{[0]} \hookrightarrow \operatorname{gr} A$. By a theorem of Radford [26], gr $A \cong R \# A_{[0]}$ as Hopf algebras, where $R=(\operatorname{gr} A)^{c o \pi}=\oplus_{n \geq 0} R(n)$ is a connected $\mathbb{N}$-graded braided Hopf algebra in ${ }_{A_{[0]}}^{A_{0]}} \mathcal{Y D}$ called the diagram of $A$. Moreover, $R(1)$ as a subspace of $\mathcal{P}(R)$ is a braided vector space called the infinitesimal braiding of $A$. If the coradical $A_{0}$ is a Hopf

2010 Mathematics Subject Classification. 16T05.

Key words and phrases. Nichols algebra; Hopf algebra; generalized lifting method.

The paper is supported by the NSFC (Grant No. 11771142) and in part by Science and Technology Commission of Shanghai Municipality (No. 18dz2271000). 
subalgebra, then the standard filtration coincides with the coradical filtration. In this case, $\operatorname{gr} A$ is coradically graded and the diagram $R$ of $A$ is strictly graded, that is, $R(0)=\mathbb{k}, R(1)=\mathcal{P}(R)$. In general, it is an open question whether the diagram $R$ is strictly graded. See [8, 3] for details. The generalized lifting method consists of the following questions (see [3]):

- Question 1. Let $C$ be a cosemisimple coalgebra and $\mathcal{S}: C \rightarrow C$ an injective anti-coalgebra morphism. Classify all Hopf algebras $L$ generated by $C$, such that $\left.S\right|_{C}=\mathcal{S}$.

- Question 2. Given $L$ as in the previous item, classify all connected graded Hopf algebras $R$ in ${ }_{L}^{L} \mathcal{Y} \mathcal{D}$.

- Question 3. Given $L$ and $R$ as in previous items, classify all liftings, that is, classify all Hopf algebras $A$ such that gr $A \cong R \sharp L$. We call $A$ a lifting of $R$ over $L$.

The motivation of this paper is [15] (also [3]). We fix two 16-dimensional Hopf algebras $H$ and $\widetilde{H}$ appearing in [11, 16] without the dual Chevalley property and study questions 2 and 3 .

The Hopf algebras $H$ and $\widetilde{H}$ defined in Definitions 3.1 and 4.1 are generated by their coradicals and have pointed duals appearing in [14]. In particular, $\widetilde{H} \cong$ $K \otimes \mathbb{k}\left[\mathbb{Z}_{2}\right]$ as Hopf algebras, where $K$ is isomorphic to the Hopf algebra $\mathcal{K}$ defined in [15, Proposition 2.1]. See subsections 3.1 and 4.1 for details.

For $H$, we determine all simple objects in ${ }_{H}^{H} \mathcal{Y D}$ by using the equivalence ${ }_{H}^{H} \mathcal{Y D} \cong$ $\mathcal{D}\left(H^{\text {cop }}\right) \mathcal{M}$ [25, Proposition 10.6.16]. Indeed, we show in Theorem 3.7 that there are 16 one-dimensional objects $\mathbb{k}_{\chi_{i, j, k}}$ with $0 \leq i, j<2,0 \leq k<4$ and 48 twodimensional objects $V_{i, j, k, \iota}$ with $(i, j, k, \iota) \in \Lambda=\{(i, j, k, \iota) \mid 0 \leq i, j<4,0 \leq$ $k, \iota<2,2 k+j \neq 2(\iota+1) \bmod 4\}$. Then we determine all finite-dimensional Nichols algebras over simple objects in ${ }_{H}^{H} \mathcal{Y} \mathcal{D}$. Finally, we calculate their liftings following the techniques in [7, 15]. We obtain the following result.

Theorem A. Let $A$ be a finite-dimensional Hopf algebra over $H$ such that the corresponding infinitesimal braiding is a simple object $V$ in ${ }_{H}^{H} \mathcal{Y D}$. Assume that the diagram of $A$ is strictly graded. Then $V$ is isomorphic either to $\mathbb{k}_{\chi_{i, j, k} \text { for }}$ $(i, j, k) \in \Lambda^{0}$ or to $V_{i, j, k, \iota}$ for $(i, j, k, \iota) \in \Lambda^{2} \cup \Lambda^{3} \cup \Lambda^{4}$, and $A$ is isomorphic either to

- $\bigwedge \mathbb{k}_{\chi_{i, j, k}} \sharp H$ for $(i, j, k) \in \Lambda^{0}$;

- $\mathcal{B}\left(V_{i, j, k, \iota}\right) \sharp H$ for $(i, j, k, \iota) \in \Lambda^{2} \cup \Lambda^{3}$;

- $\mathfrak{C}_{i, j, k, \iota}^{4}(\mu)$ for $\mu \in \mathbb{k}$ and $(i, j, k, \iota) \in \Lambda^{4}$.

The sets $\Lambda^{0}, \Lambda^{2}, \Lambda^{3}, \Lambda^{4}$ as subsets of $\Lambda$ are introduced in Lemma 3.13 and Proposition 3.14 and $\left|\Lambda^{2}\right|-4=\left|\Lambda^{3}\right|-8=\left|\Lambda^{4}\right|-8=0$. It turns out that $\mathcal{B}\left(\mathbb{k}_{\chi_{i, j, k}}\right)$ is an exterior algebra for $(i, j, k) \in \Lambda^{0}$, and $\mathcal{B}\left(V_{i, j, k, \iota}\right)$ is isomorphic as an algebra to a quantum plane of dimension 4 or 8 for $(i, j, k, \iota) \in \Lambda^{2}$ or $\Lambda^{3} \cup \Lambda^{4}$, respectively. These Nichols algebras appearing firstly in [23] were also described in [4] as special kinds. As stated in [4, they are not of diagonal type.

The Hopf algebras $\bigwedge \mathbb{k}_{\chi_{i, j, k}} \sharp H$ with $(i, j, k) \in \Lambda^{0}, \mathcal{B}\left(V_{i, j, k, \iota}\right) \sharp H$ with $(i, j, k, \iota) \in$ $\Lambda^{2}$ or with $(i, j, k, \iota) \in \Lambda^{3} \cup \Lambda^{4}$ are the duals of pointed Hopf algebras of dimension 
32,64 or 128, respectively. The Hopf algebras $\mathfrak{C}_{i, j, k, \iota}^{4}(\mu)$ depending on the parameters $\mu \in \mathbb{k}$ and $(i, j, k, \iota) \in \Lambda^{4}$ are introduced in Definitions 3.20 and 3.22 They have dimension 128 with non-pointed duals and constitute new examples of Hopf algebras without the dual Chevalley property except for $\mu=0$.

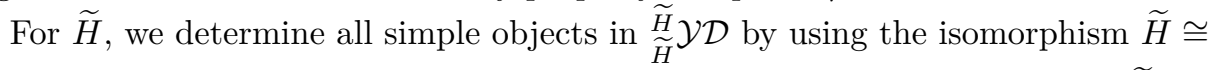
$K \otimes \mathbb{k}\left[\mathbb{Z}_{2}\right]$. We show that there are 16 one-dimensional objects $\mathbb{k}_{\lambda_{i, j, k}}$ in $\underset{\widetilde{H}}{\widetilde{\widetilde{H}}} \mathcal{Y} \mathcal{D}$ for $i, j \in \mathbb{I}_{0,1}, k \in \mathbb{I}_{0,3}$, and 48 two-dimensional simple objects $W_{i, j, k, \iota}$ in $\underset{\widetilde{H}}{\widetilde{Y} \mathcal{D}}$ for $(i, j, k, \iota) \in \Omega=\left\{(i, j, k, \iota) \mid i, j \in \mathbb{I}_{0,3}, k, \iota \in \mathbb{I}_{0,1}, 2 i \neq j \bmod 4\right\}$. Then

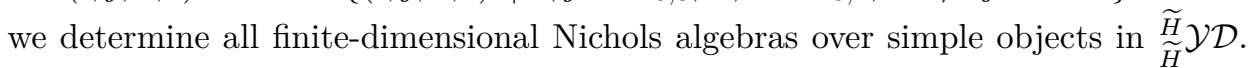
Finally, we calculate their liftings. We obtain the following result.

Theorem B. Let $A$ be a finite-dimensional Hopf algebra over $\widetilde{H}$ such that the

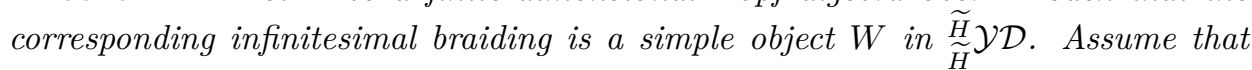
the diagram of $A$ is strictly graded. Then $W$ is isomorphic either to $\mathbb{k}_{\lambda_{i, j, k}}$ for $(i, j, k) \in \Omega^{0}$ or to $W_{i, j, k, \iota}$ for $(i, j, k, \iota) \in \Omega^{1} \cup \Omega^{2} \cup \Omega^{3}$ and $A$ is isomorphic either to

- $\bigwedge \mathrm{k}_{\lambda_{i, j, k}} \sharp \widetilde{H}$ for $(i, j, k) \in \Omega^{0}$;

- $\mathcal{B}\left(W_{i, j, k, \iota}\right) \sharp \widetilde{H}$ for $(i, j, k, \iota) \in \Omega^{1} \cup \Omega^{2}$;

- $\Omega_{i, j, k, \iota}^{3}(\mu)$ for $\mu \in \mathbb{k}$ and $(i, j, k, \iota) \in \Omega^{3}$.

The set $\Omega^{i}$ for $0 \leq i \leq 3$ as a subset of $\Omega$ is introduced in Lemma 4.9 or Proposition 4.10 and $\left|\Omega^{1}\right|-4=\left|\Omega^{2}\right|-8=\left|\Omega^{3}\right|-8=0$. It turns out that $\mathcal{B}\left(\mathbb{k}_{\lambda_{i, j, k}}\right)$ is an exterior algebra for $(i, j, k) \in \Omega^{0}, \operatorname{dim} \mathcal{B}\left(W_{i, j, k, \iota}\right)=4$ for $(i, j, k, \iota) \in \Omega^{1}$ and $\operatorname{dim} \mathcal{B}\left(W_{i, j, k, \iota}\right)=8$ for $(i, j, k, \iota) \in \Omega^{2} \cup \Omega^{3}$. These 8-dimensional Nichols algebras were firstly introduced in [15] and these 4-dimensional Nichols algebras did not appear in [15] but have already appeared in [4]. They are isomorphic to quantum planes as algebras but not as coalgebras since they are not of diagonal type.

For $(i, j, k) \in \Omega^{0},(i, j, k, \iota) \in \Omega^{1}$ and $(i, j, k, \iota) \in \Omega^{2} \cup \Omega^{3}$, the Hopf algebras $\bigwedge \mathrm{k}_{\lambda_{i, j, k}} \sharp \widetilde{H}$ and $\mathcal{B}\left(W_{i, j, k, \iota}\right) \sharp \widetilde{H}$ are the duals of pointed Hopf algebras of dimension 32,64 and 128, respectively. In particular, $\mathcal{B}\left(W_{2, j, 0,0}\right) \sharp \widetilde{H} \cong \mathcal{B}\left(W_{2, j, 0,0}\right) \sharp K \otimes \mathbb{k}\left[\mathbb{Z}_{2}\right]$ as Hopf algebras for $j \in\{1,3\}$. For $(i, j, k, \iota) \in \Omega^{3}$, the Hopf algebra $\Omega_{i, j, k, \iota}^{3}(\mu)$ depending on the parameter $\mu \in \mathbb{k}$ is introduced in Definition 4.16. Note that $\Omega_{i, j, k, \iota}^{3}(0) \cong \mathcal{B}\left(W_{i, j, k, \iota}\right) \sharp \widetilde{H}$ for $(i, j, k, \iota) \in \Omega^{3}$ and $\Omega_{3, j, 0,0}^{3}(\mu) \cong \mathfrak{A}_{3, j}(\mu) \otimes \mathbb{k}\left[\mathbb{Z}_{2}\right]$ as Hopf algebras, where $j \in\{1,3\}$ and $\mathfrak{A}_{3, j}(\mu)$ is given in [15, Definitions 5.4/5.6]. The Hopf algebras $\Omega_{i, j, k, \iota}^{3}(\mu)$ with $(k, \iota, \mu) \neq(0,0,0)$ are not isomorphic to the tensor product Hopf algebra of a Hopf algebra of dimension 64 and $\mathbb{k}\left[\mathbb{Z}_{2}\right]$, and do not have the dual Chevalley property with non-pointed duals. To the best of our knowledge, they constitute new examples of Hopf algebras of dimension 128.

The paper is organized as follows. In section 2, we recall some basic knowledge and notations of Yetter-Drinfeld modules, Nichols algebras and Radford biproduct. In section 3 we determine all finite-dimensional Nichols algebras over simple objects in ${ }_{H}^{H} \mathcal{Y D}$ and their liftings. We first describe the structures of $H$ and the Drinfeld double $\mathcal{D}:=\mathcal{D}\left(H^{c o p}\right)$. Next, we determine all simple $\mathcal{D}$-modules and describe 
simple objects in ${ }_{H}^{H} \mathcal{Y D}$ by using the equivalence ${ }_{H}^{H} \mathcal{Y D} \cong{ }_{\mathcal{D}} \mathcal{M}$. Then we describe the braidings and determine all finite-dimensional Nichols algebras over simple objects in ${ }_{H}^{H} \mathcal{Y} \mathcal{D}$. Finally, we calculate the liftings of all finite-dimensional Nichols algebras and prove Theorem A. In section 4, we determine all finite-dimensional Nichols

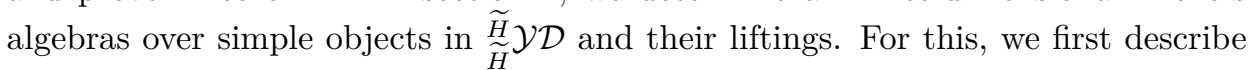
the structure of $\widetilde{H}$ and determine simple objects in $\underset{H}{\widetilde{H}} \mathcal{Y} \mathcal{D}$ by using the isomorphism $\widetilde{H} \cong \mathcal{K} \otimes \mathbb{k}\left[\mathbb{Z}_{2}\right]$. Then we describe the braidings and determine all finite-dimensional Nichols algebras over simple objects in $\underset{\widetilde{H}}{\widetilde{Y}} \mathcal{D}$. Finally, we calculate the liftings of all finite-dimensional Nichols algebras and prove Theorem $B$.

\section{PReliminaries}

Conventions. Throughout the paper, our ground field $\mathbb{k}$ is an algebraically closed field of characteristic zero. We denote by $\xi$ a primitive 4 th root of unity. Our references for Hopf algebra theory are [25, 27].

The notation for a Hopf algebra $H$ over $\mathbb{k}$ is standard: $\Delta, \epsilon$, and $S$ denote the comultiplication, the counit and the antipode. We use Sweedler's notation for the comultiplication and coaction; for example, for any $h \in H, \Delta(h)=h_{(1)} \otimes h_{(2)}$, $\Delta^{(n)}=\left(\Delta \otimes i d^{\otimes n}\right) \Delta^{(n-1)}$. We denote by $H^{o p}$ the Hopf algebra with the opposite multiplication, by $H^{c o p}$ the Hopf algebra with the opposite comultiplication, and by $H^{\text {bop }}$ the Hopf algebra $H^{o p c o p}$. Denote by $\mathcal{G}(H)$ the set of group-like elements of $H$. For any $g, h \in \mathcal{G}(H), \mathcal{P}_{g, h}(H)=\{x \in H \mid \Delta(x)=x \otimes g+h \otimes x\}$. In particular, the linear space $\mathcal{P}(H):=\mathcal{P}_{1,1}(H)$ is called the set of primitive elements.

Given two (braided monoidal) categories $\mathfrak{C}$ and $\mathfrak{D}$, denote by $\mathfrak{C} \cong \mathfrak{D}$ the (braided monoidal) equivalence between $\mathfrak{C}$ and $\mathfrak{D}$. Given $n \geq 0$, we denote $\mathbb{Z}_{n}=\mathbb{Z} / n \mathbb{Z}$ and $\mathbb{I}_{0, n}=\{0,1, \ldots, n\}$. In particular, the operations $i j$ and $i \pm j$ are considered modulo $n+1$ for $i, j \in \mathbb{I}_{0, n}$ when not specified.

2.1. Yetter-Drinfeld modules and Nichols algebras. Let $H$ be a Hopf algebra with bijective antipode. A left Yetter-Drinfeld module $M$ over $H$ is a left $H$-module $(M, \cdot)$ and a left $H$-comodule $(M, \delta)$ satisfying

$$
\delta(h \cdot v)=h_{(1)} v_{(-1)} S\left(h_{(3)}\right) \otimes h_{(2)} \cdot v_{(0)}, \quad \forall v \in V, h \in H .
$$

Let ${ }_{H}^{H} \mathcal{Y D}$ be the category of Yetter-Drinfeld modules over $H .{ }_{H}^{H} \mathcal{Y D}$ is braided monoidal. For $V, W \in{ }_{H}^{H} \mathcal{Y} \mathcal{D}$, the braiding $c_{V, W}$ is given by

$$
c_{V, W}: V \otimes W \mapsto W \otimes V, \quad v \otimes w \mapsto v_{(-1)} \cdot w \otimes v_{(0)}, \quad \forall v \in V, w \in W .
$$

Moreover, ${ }_{H}^{H} \mathcal{Y D}$ is rigid. Denote by $V^{*}$ the left dual defined by

$$
\langle h \cdot f, v\rangle=\langle f, S(h) v\rangle, \quad f_{(-1)}\left\langle f_{(0)}, v\right\rangle=S^{-1}\left(v_{(-1)}\right)\left\langle f, v_{(0)}\right\rangle .
$$

Assume that $H$ is a finite-dimensional Hopf algebra. Then ${ }_{H^{*}}^{H^{*}} \mathcal{Y D}$ is braided equivalent to ${ }_{H}^{H} \mathcal{Y D}$, see [5, 2.2.1]. Let $\left\{h_{i}\right\}_{i \in \mathbb{I}_{0, n}}$ and $\left\{h^{i}\right\}_{i \in \mathbb{I}_{0, n}}$ be the dual bases 
of $H$ and $H^{*}$. If $V \in{ }_{H}^{H} \mathcal{Y D}$, then $V \in{ }_{H^{*}}^{H^{*}} \mathcal{Y D}$ with the Yetter-Drinfeld module structure given by

$$
f \cdot v=f\left(S\left(v_{(-1)}\right)\right) v_{(0)}, \quad \delta(v)=\sum_{i} S^{-1}\left(h^{i}\right) \otimes h_{i} \cdot v, \quad \forall v \in V, f \in H^{*} .
$$

Definition 2.1 ([8, Definition 2.1]). Let $H$ be a Hopf algebra and $V \in{ }_{H}^{H} \mathcal{Y D}$. A braided graded Hopf algebra $R=\oplus_{n \geq 0} R(n)$ in ${ }_{H}^{H} \mathcal{Y D}$ is called a Nichols algebra over $V$ if

$$
R(0)=\mathbb{k}, \quad R(1)=V, \quad R \text { is generated as an algebra by } R(1), \quad \mathcal{P}(R)=V .
$$

Let $V \in{ }_{H}^{H} \mathcal{Y D}$, then the Nichols algebra $\mathcal{B}(V)$ over $V$ is unique up to isomorphism and isomorphic to $T(V) / I(V)$, where $I(V) \subset T(V)$ is the largest $\mathbb{N}$-graded ideal and coideal in ${ }_{H}^{H} \mathcal{Y D}$ such that $I(V) \cap V=0$.

Remark 2.2. Let $(V, c)$ be a braided vector space, that is, c: $V \otimes V \mapsto V \otimes V$ is a linear isomorphism satisfying the braid equation $(c \otimes i d)(i d \otimes c)(c \otimes i d)=$ $(i d \otimes c)(c \otimes i d)(i d \otimes c)$. As well-known, $\mathcal{B}(V)$ as a coalgebra and an algebra depends only on $(V, c)$. Let $(W, c)$ be a vector subspace of $V$ such that $c(W \otimes W) \subset W \otimes W$. Then $\operatorname{dim} \mathcal{B}(V)=\infty$ if $\operatorname{dim} \mathcal{B}(W)=\infty$. See [17, 8] for details.

Nichols algebras play a key role in the classification of pointed Hopf algebras. We close this subsection by giving the explicit relation between $V$ and $V^{*}$ in ${ }_{H}^{H} \mathcal{Y} \mathcal{D}$.

Proposition 2.3 ([5, Proposition 3.2.30]). Let $V$ be an object in ${ }_{H}^{H} \mathcal{Y D}$. If $\mathcal{B}(V)$ is finite-dimensional, then $\mathcal{B}\left(V^{*}\right) \cong \mathcal{B}(V)^{* \text { bop }}$.

2.2. Bosonization and Hopf algebras with a projection. Let $R$ be a Hopf algebra in ${ }_{H}^{H} \mathcal{Y} \mathcal{D}$. We write $\Delta_{R}(r)=r^{(1)} \otimes r^{(2)}$ to avoid confusions. The bosonization $R \sharp H$ is defined as follows: $R \sharp H=R \otimes H$ as a vector space, and the multiplication and comultiplication are given by the smash product and smash-coproduct, respectively:

$$
(r \sharp g)(s \sharp h)=r\left(g_{(1)} \cdot s\right) \sharp g_{(2)} h, \quad \Delta(r \sharp g)=r^{(1)} \sharp\left(r^{(2)}\right)_{(-1)} g_{(1)} \otimes\left(r^{(2)}\right)_{(0)} \sharp g_{(2)} .
$$

Clearly, the map $\iota: H \rightarrow R \sharp H, h \mapsto 1 \sharp h, \forall h \in H$, is injective and the map $\pi: R \sharp H \rightarrow H, r \sharp h \mapsto \epsilon_{R}(r) h, \forall r \in R, h \in H$, is surjective such that $\pi \circ \iota=i d_{H}$. Moreover, $R=(R \sharp H)^{c o H}=\{x \in R \sharp H \mid(\mathrm{id} \otimes \pi) \Delta(x)=x \otimes 1\}$.

Conversely, if $A$ is a Hopf algebra with bijective antipode and $\pi: A \rightarrow H$ is a bialgebra morphism admitting a bialgebra section $\iota: H \rightarrow A$ such that $\pi \circ \iota=\mathrm{id}_{H}$, then $A \simeq R \sharp H$, where $R=A^{c o H}$ is a Hopf algebra in ${ }_{H}^{H} \mathcal{Y} \mathcal{D}$. See [26] for details.

\section{On finite-Dimensional Hopf algebras over $H$}

In this section, we determine all finite-dimensional Nichols algebras over simple objects in ${ }_{H}^{H} \mathcal{Y D}$ and their liftings. These Nichols algebras have already appeared in [15, 4] and consist of 2-dimensional exterior algebras, 4- and 8-dimensional algebras with non-diagonal braidings [29, 15]. The bosonizations of these Nichols algebras are finite-dimensional Hopf algebras over $H$ without the dual Chevalley property. Moreover, the non-trivial liftings of these Nichols algebras constitute new examples of Hopf algebras of dimension 128 without the dual Chevalley property. 
3.1. The Hopf algebra $H$ and its Drinfeld double. We firstly describe the Hopf algebra $H$, which already appeared in [11, 16] and is generated by a simple subcoalgebra $C=\mathbb{k}\{a, b, c, d\}$ as follows.

Definition 3.1. $H$ as an algebra is generated by $a, b, c, d$ satisfying the relations

$$
\begin{gathered}
a^{4}=1, \quad b^{2}=0, \quad c^{2}=0, \quad d^{4}=1, \quad a^{2} d^{2}=1, \quad a d=d a, \quad b c=0=c b, \\
a b=\xi b a, \quad a c=\xi c a, \quad b d=\xi d b, \quad c d=\xi d c, \quad b d=c a, \quad b a=c d,
\end{gathered}
$$

and as a coalgebra is given by

$$
\begin{gathered}
\Delta(a)=a \otimes a+b \otimes c, \quad \Delta(b)=a \otimes b+b \otimes d, \quad \Delta(c)=c \otimes a+d \otimes c, \\
\Delta(d)=d \otimes d+c \otimes b, \quad \epsilon(a)=1, \quad \epsilon(b)=0, \quad \epsilon(c)=0, \quad \epsilon(d)=1,
\end{gathered}
$$

and its antipode is given by $S(a)=a^{3}, S(b)=\xi c a^{2}, S(c)=\xi^{3} b a^{2}, S(d)=d^{3}$.

Remark 3.2. (1) $\mathcal{G}(H)=\mathbb{k}\left\{1, a^{2}, d a, d a^{3}\right\}, \mathcal{P}_{1, d a^{3}}(H)=\mathbb{k}\left\{1-d a^{3}, c a^{3}\right\}$, $\mathcal{P}_{1, g}(H)=\mathbb{k}\{1-g\}$ for $g \in \mathbb{k}\left\{a^{2}, d a\right\}$ and a linear basis of $H$ is given by $\left\{a^{i}, b a^{i}, c a^{i}, d a^{i}, i \in \mathbb{I}_{0,3}\right\}$.

(2) Denote by $\left\{\left(a^{i}\right)^{*},\left(b a^{i}\right)^{*},\left(c a^{i}\right)^{*},\left(d a^{i}\right)^{*}, i \in \mathbb{I}_{0,3}\right\}$ the basis of the dual Hopf algebra $H^{*}$. Let

$$
\widetilde{x}=\sum_{i=0}^{3}\left(b a^{i}\right)^{*}+\left(c a^{i}\right)^{*}, \quad \widetilde{g}=\sum_{i=0}^{3} \xi^{i}\left(a^{i}\right)^{*}+\xi^{i+1}\left(d a^{i}\right)^{*}, \quad \widetilde{h}=\sum_{i=0}^{3}\left(a^{i}\right)^{*}-\left(d a^{i}\right)^{*} .
$$

Then using the multiplication table induced by the relations of $H$, we have

$$
\begin{gathered}
\widetilde{g}^{4}=1, \quad \widetilde{h}^{2}=1, \quad \widetilde{h} \widetilde{g}=\widetilde{g} \tilde{h}, \quad \widetilde{g} \widetilde{x}=\widetilde{x} \widetilde{g}, \quad \widetilde{h} \widetilde{x}=-\widetilde{x} \tilde{h}, \\
\Delta(\widetilde{x})=\widetilde{x} \otimes \epsilon+\widetilde{g} \tilde{h} \otimes \widetilde{x}, \quad \Delta(\widetilde{g})=\widetilde{g} \otimes \widetilde{g}, \quad \Delta(\widetilde{h})=\widetilde{h} \otimes \widetilde{h} .
\end{gathered}
$$

In particular, $\mathcal{G}\left(H^{*}\right) \cong \mathbb{Z}_{4} \times \mathbb{Z}_{2}$ with the generators $\widetilde{g}$ and $\widetilde{h}$.

(3) Let $\mathcal{A}$ be the Hopf algebra defined by $\mathcal{A}:=\langle g, h, x| g^{4}=1, h^{2}=1, h g=$ $\left.g h, h x=-x h, g x=x g, x^{2}=1-g^{2}\right\rangle ; \Delta(g)=g \otimes g, \Delta(h)=h \otimes h, \Delta(x)=$ $x \otimes 1+g h \otimes x$. It is listed in [14, section 2.5]. Clearly, $\mathcal{G}(\mathcal{A}) \cong \mathbb{Z}_{4} \times \mathbb{Z}_{2}$ and $\left\{g^{j}, g^{j} h, g^{j} x, g^{j} h x\right\}_{0 \leq j<4}$ is a linear basis of $\mathcal{A}$. Moreover, $\mathcal{A} \cong H^{*}$ and the Hopf algebra isomorphism $\psi: \mathcal{A} \mapsto H^{*}$ is given by

$$
\begin{aligned}
& \psi\left(g^{j}\right)=\sum_{i=0}^{3} \xi^{i j}\left(a^{i}\right)^{*}+\xi^{i j+j}\left(d a^{i}\right)^{*}, \quad \psi\left(g^{j} h\right)=\sum_{i=0}^{3} \xi^{i j}\left(a^{i}\right)^{*}-\xi^{i j+j}\left(d a^{i}\right)^{*}, \\
& \psi\left(g^{j} x\right)=\sum_{i=0}^{3} \sqrt{2} \xi^{i j+j}\left(\left(b a^{i}\right)^{*}+\left(c a^{i}\right)^{*}\right), \quad \psi\left(g^{j} h x\right)=\sum_{i=0}^{3} \sqrt{2} \xi^{i j+j}\left(\left(b a^{i}\right)^{*}-\left(c a^{i}\right)^{*}\right) .
\end{aligned}
$$

Now we describe the Drinfeld double $\mathcal{D}:=\mathcal{D}\left(H^{c o p}\right)$ of $H^{c o p}$. Recall that $\mathcal{D}(H) \cong$ $H^{* c o p} \otimes H$ is a Hopf algebra with the tensor product coalgebra structure and the algebra structure given by $(p \otimes a)(q \otimes b)=p\left\langle q_{(3)}, a_{(1)}\right\rangle q_{(2)} \otimes a_{(2)}\left\langle q_{(1)}, S^{-1}\left(a_{(3)}\right)\right\rangle b$. 
Proposition 3.3. $\mathcal{D}:=\mathcal{D}\left(H^{c o p}\right)$ as a coalgebra is isomorphic to $\mathcal{A}^{b o p} \otimes H^{c o p}$, and as an algebra is generated by the elements $g, h, x, a, b, c, d$ satisfying the relations in $H^{\text {cop }}$, the relations in $\mathcal{A}^{\text {bop }}$ and

$$
\begin{gathered}
a g=g a, \quad a h=h a, \quad d g=g d, \quad d h=h d, \quad b g=g b, \quad c g=g c, \\
b h=-h b, \quad a x+\xi x a=\sqrt{2} \xi(c-g h b), \quad d x-\xi x d=\sqrt{2} \xi(g h c-b), \\
c h=-h c, \quad b x+\xi x b=\sqrt{2} \xi(d-g h a), \quad c x-\xi x c=\sqrt{2} \xi(g h d-a) .
\end{gathered}
$$

Proof. After a direct computation, we have that

$$
\begin{aligned}
& \Delta_{\mathcal{A}^{\text {bop }}}^{2}(g)=g \otimes g \otimes g, \quad \Delta_{\mathcal{A}^{\text {bop }}}^{2}(h)=h \otimes h \otimes h, \\
& \Delta_{\mathcal{A}^{\text {bop }}}^{2}(x)=1 \otimes 1 \otimes x+1 \otimes x \otimes g h+x \otimes g h \otimes g h, \\
& \Delta_{H^{\text {cop }}}^{2}(a)=a \otimes a \otimes a+a \otimes c \otimes b+c \otimes b \otimes a+c \otimes d \otimes b, \\
& \Delta_{H^{\text {cop }}}^{2}(b)=b \otimes a \otimes a+b \otimes c \otimes b+d \otimes b \otimes a+d \otimes d \otimes b, \\
& \Delta_{H^{\text {cop }}}^{2}(c)=a \otimes a \otimes c+a \otimes c \otimes d+c \otimes d \otimes d+c \otimes b \otimes c, \\
& \Delta_{H^{\text {cop }}}^{2}(d)=d \otimes d \otimes d+d \otimes b \otimes c+b \otimes a \otimes c+b \otimes c \otimes d .
\end{aligned}
$$

It follows that

$$
\begin{aligned}
a g & =\langle g, a\rangle g a\langle g, S(a)\rangle=g a, \quad a h=\langle h, a\rangle h a\langle h, S(a)\rangle=h a, \\
d g & =\langle g, d\rangle g d\langle g, S(d)\rangle=g d, \quad d h=\langle h, d\rangle h d\langle h, S(d)\rangle=h d, \\
b g & =\langle g, d\rangle g b\langle g, S(a)\rangle=g b, \quad b h=\langle h, d\rangle h b\langle h, S(a)\rangle=-h b, \\
c g & =\langle g, a\rangle g c\langle g, S(d)\rangle=g c, \quad c h=\langle h, a\rangle h c\langle h, S(d)\rangle=-h c, \\
a x & =\langle 1, a\rangle c\langle x, S(b)\rangle+\langle 1, a\rangle x a\langle g h, S(a)\rangle+\langle x, c\rangle g h b\langle g h, S(a)\rangle \\
& =\sqrt{2} \xi c-\xi x a-\sqrt{2} \xi g h b, \\
d x & =\langle 1, d\rangle b\langle x, S(c)\rangle+\langle 1, d\rangle x d\langle g h, S(d)\rangle+\langle x, b\rangle g h c\langle g h, S(d)\rangle \\
& =\sqrt{2} \xi^{3} b+\xi x d+\sqrt{2} \xi g h c, \\
b x & =\langle 1, d\rangle d\langle x, S(b)\rangle+\langle 1, d\rangle x b\langle g h, S(a)\rangle+\langle x, b\rangle g h a\langle g h, S(a)\rangle \\
& =\sqrt{2} \xi d-\xi x b-\sqrt{2} \xi g h a, \\
c x & =\langle 1, a\rangle a\langle x, S(c)\rangle+\langle 1, a\rangle x c\langle g h, S(d)\rangle+\langle x, c\rangle g h d\langle g h, S(d)\rangle \\
& =\sqrt{2} \xi^{3} a+\xi x c+\sqrt{2} \xi g h d .
\end{aligned}
$$

3.2. The representations of $\mathcal{D}$. We compute simple $\mathcal{D}$-modules. We begin this subsection by describing the one-dimensional $\mathcal{D}$-modules.

Lemma 3.4. There are 16 non-isomorphic one-dimensional simple modules $\mathbb{k}_{\chi_{i, j, k}}$ given by the characters $\chi_{i, j, k}$ with $i, j \in \mathbb{I}_{0,1}, k \in \mathbb{I}_{0,3}$, where

$$
\begin{gathered}
\chi_{i, j, k}(g)=(-1)^{i}, \quad \chi_{i, j, k}(h)=(-1)^{j}, \quad \chi_{i, j, k}(x)=0, \\
\chi_{i, j, k}(a)=\xi^{k}, \quad \chi_{i, j, k}(b)=0, \quad \chi_{i, j, k}(c)=0, \quad \chi_{i, j, k}(d)=(-1)^{i}(-1)^{j} \xi^{k} .
\end{gathered}
$$

Moreover, any one-dimensional $\mathcal{D}$-module is isomorphic to $\mathbb{k}_{\chi_{i, j, k}}$ for some $i, j \in$ $\mathbb{I}_{0,1}, k \in \mathbb{I}_{0,3}$. 
Proof. It is clear that these modules $\mathrm{k}_{\chi_{i, j, k}}$ are pairwise non-isomorphic. Let $\chi \in$ $G\left(\mathcal{D}^{*}\right)=\operatorname{hom}(\mathcal{D}, \mathrm{k})$. Since $a^{4}=g^{4}=d^{4}=h^{2}=1$, we have $\chi(a)^{4}=\chi(g)^{4}=$ $\chi(d)^{4}=\chi(h)^{2}=1$. Since $b^{2}=c^{2}=h x+x h=0$, it follows that $\chi(b)=\chi(x)=$ $\chi(c)=0$. Then the relation $x^{2}=1-g^{2}$ yields $\chi(g)^{2}=1$. From the relation $b x+\xi x b=\sqrt{2} \xi(d-g h a)$, we have $\chi(d)=\chi(g) \chi(h) \chi(a)$. Thus $\chi$ is completely determined by $\chi(a), \chi(g)$ and $\chi(h)$. Let $\chi(a)=\xi^{k}, \chi(g)=(-1)^{i}, \chi(h)=(-1)^{j}$ for some $i, j \in \mathbb{I}_{0,1}, k \in \mathbb{I}_{0,3}$. Then $\chi=\chi_{i, j, k}$. The lemma is proved.

Next, we describe two-dimensional simple $\mathcal{D}$-modules. For this, consider the finite set given by

$\Lambda=\left\{(i, j, k, \iota) \in \mathbb{N} \times \mathbb{N} \times \mathbb{N} \times \mathbb{N} \mid i, j \in \mathbb{I}_{0,3}, k, \iota \in \mathbb{I}_{0,1}, 2 k+j \neq 2(\iota+1) \bmod 4\right\}$.

A direct calculation shows that $|\Lambda|=48$.

Lemma 3.5. For any 4 -tuple $(i, j, k, \iota) \in \Lambda$, there exists a simple left $\mathcal{D}$-module $V_{i, j, k, \iota}$ of dimension 2 with the action on a fixed basis given by

$$
\begin{aligned}
& {[a]=\left(\begin{array}{cc}
-(-1)^{\iota} \xi^{i} & 0 \\
0 & (-1)^{\iota} \xi^{i+1}
\end{array}\right), \quad[d]=\left(\begin{array}{cc}
\xi^{i} & 0 \\
0 & \xi^{i+1}
\end{array}\right), \quad[b]=\left(\begin{array}{cc}
0 & (-1)^{\iota} \\
0 & 0
\end{array}\right),} \\
& {[c]=\left(\begin{array}{cc}
0 & 1 \\
0 & 0
\end{array}\right), \quad[g]=\left(\begin{array}{cc}
\xi^{j} & 0 \\
0 & \xi^{j}
\end{array}\right), \quad[h]=\left(\begin{array}{cc}
(-1)^{k} & 0 \\
0 & (-1)^{k+1}
\end{array}\right),} \\
& {[x]=\left(\begin{array}{cc}
0 & \frac{\sqrt{2}}{2} \xi^{3 i+1}\left(\xi^{j}(-1)^{k}-(-1)^{\iota}\right) \\
\sqrt{2} \xi^{i+1}\left(\xi^{j}(-1)^{k}+(-1)^{\iota}\right) & 0
\end{array}\right) .}
\end{aligned}
$$

Moreover, any simple $\mathcal{D}$-module of dimension 2 is isomorphic to $V_{i, j, k, \iota}$ for some $(i, j, k, \iota) \in \Lambda$ and $V_{i, j, k, \iota} \cong V_{p, q, r, \kappa}$ if and only if $(i, j, k, \iota)=(p, q, r, \kappa)$.

Proof. Since the elements $g, h, a, d$ commute with each other and $g^{4}=h^{2}=a^{4}=$ $d^{4}=1$, the matrices defining $\mathcal{D}$-action on $V$ can be of the form

$$
\begin{array}{ll}
{[g]=\left(\begin{array}{cc}
g_{1} & 0 \\
0 & g_{2}
\end{array}\right), \quad[h]=\left(\begin{array}{cc}
h_{1} & 0 \\
0 & h_{2}
\end{array}\right), \quad[x]=\left(\begin{array}{ll}
x_{1} & x_{2} \\
x_{3} & x_{4}
\end{array}\right), \quad[a]=\left(\begin{array}{cc}
a_{1} & 0 \\
0 & a_{2}
\end{array}\right),} \\
{[d]=\left(\begin{array}{cc}
d_{1} & 0 \\
0 & d_{2}
\end{array}\right), \quad[b]=\left(\begin{array}{ll}
b_{1} & b_{2} \\
b_{3} & b_{4}
\end{array}\right), \quad[c]=\left(\begin{array}{ll}
c_{1} & c_{2} \\
c_{3} & c_{4}
\end{array}\right),}
\end{array}
$$

where $a_{1}^{4}=a_{2}^{4}=d_{1}^{4}=d_{2}^{4}=g_{1}^{4}=g_{2}^{4}=h_{1}^{2}=h_{2}^{2}=1$. Since $x h+h x=b h+h b=$ $c h+h c=0$, it follows that $x_{1}=x_{4}=b_{1}=b_{4}=c_{1}=c_{4}=0,\left(h_{1}+h_{2}\right) x_{2}=0=$ $\left(h_{1}+h_{2}\right) x_{3},\left(h_{1}+h_{2}\right) b_{2}=0=\left(h_{1}+h_{2}\right) b_{3}$ and $\left(h_{1}+h_{2}\right) c_{2}=0=\left(h_{1}+h_{2}\right) c_{3}$.

If $h_{1}+h_{2} \neq 0$, then $[x],[b],[c]$ are zero matrices and hence $V$ is not simple $\mathcal{D}$-module, a contradiction. Therefore, we have $h_{1}=-h_{2}$. Similarly, the relations $g x=x g, b g=g b$ and $c g=g c$ yield $g_{1}=g_{2}$.

Since $b^{2}=0=c^{2}$ and $b c=0=c b$, it follows that $b_{2} b_{3}=c_{2} c_{3}=b_{2} c_{3}=b_{3} c_{2}=$ $c_{2} b_{3}=c_{3} b_{2}=0$. By permuting the elements of the basis, we may assume that $b_{3}=$ $0=c_{3}$. From the relations $a x+\xi x a=\sqrt{2} \xi(c-g h b)$ and $d x-\xi x d=\sqrt{2} \xi(g h c-b)$,

$$
\begin{array}{ll}
a_{1} x_{2}+\xi a_{2} x_{2}=\sqrt{2} \xi\left(c_{2}-g_{1} h_{1} b_{2}\right), & a_{2} x_{3}+\xi a_{1} x_{3}=\sqrt{2} \xi\left(c_{3}-g_{2} h_{2} b_{3}\right), \\
d_{1} x_{2}-\xi d_{2} x_{2}=\sqrt{2} \xi\left(g_{1} h_{1} c_{2}-b_{2}\right), & d_{2} x_{3}-\xi d_{1} x_{3}=\sqrt{2} \xi\left(g_{2} h_{2} c_{3}-b_{3}\right) .
\end{array}
$$


Suppose that $b_{2}=0=c_{2}$. Then it is clear that $V$ is simple if and only if $x_{2} x_{3} \neq 0$. By (8), we have that $a_{1}+\xi a_{2}=0=a_{2}+\xi a_{1}$ and hence $a_{1}=0=a_{2}$, a contradiction. We may also assume that $c_{2}=1$.

Since $a b=\xi b a, a c=\xi c a, b d=\xi d b$ and $c d=\xi d c$, it follows that $a_{1}-\xi a_{2}=0=$ $d_{2}-\xi d_{1}$. By the relations $b d=c a$ and $b a=c d$, we have $b_{2}^{2}-1=0=a_{2}-b_{2} d_{2}$.

From the relations $b x+\xi x b=\sqrt{2} \xi(d-g h a)$ and $c x-\xi x c=\sqrt{2} \xi(g h d-a)$,

$$
\begin{array}{ll}
b_{2} x_{3}+\xi b_{3} x_{2}=\sqrt{2} \xi\left(d_{1}-g_{1} h_{1} a_{1}\right), & b_{3} x_{2}+\xi b_{2} x_{3}=\sqrt{2} \xi\left(d_{2}-g_{2} h_{2} a_{2}\right), \\
c_{2} x_{3}-\xi c_{3} x_{2}=\sqrt{2} \xi\left(g_{1} h_{1} d_{1}-a_{1}\right), & c_{3} x_{2}-\xi c_{2} x_{3}=\sqrt{2} \xi\left(g_{2} h_{2} d_{2}-a_{2}\right),
\end{array}
$$

which implies that $x_{3}=\sqrt{2} \xi d_{1}\left(b_{2}+g_{1} h_{1}\right)$. By (8), we have $x_{2}=\frac{\sqrt{2}}{2} \xi d_{1}^{3}\left(g_{1} h_{1}-b_{2}\right)$. From the relations $x^{2}=1-g^{2}$ and $a^{2} d^{2}=1$, we have $x_{2} x_{3}=1-g_{1}^{2}$ and $a_{1}^{2} d_{1}^{2}=$ $1=a_{2}^{2} d_{2}^{2}$. Indeed, $a_{2}=b_{2} d_{2}, a_{1}=\xi a_{2}, d_{2}=\xi d_{1}, a_{1}=-b_{2} d_{1}$ and hence the relations $a_{1}^{2} d_{1}^{2}=1=a_{2}^{2} d_{2}^{2}$ hold. Moreover, it follows by a direct computation that the relation $x_{2} x_{3}=1-g_{1}^{2}$ holds.

From the discussion above, the matrices defining the action on $\mathrm{V}$ are of the form

$$
\begin{aligned}
& {[a]=\left(\begin{array}{cc}
-\lambda_{4} \lambda_{1} & 0 \\
0 & \xi \lambda_{4} \lambda_{1}
\end{array}\right), \quad[d]=\left(\begin{array}{cc}
\lambda_{1} & 0 \\
0 & \xi \lambda_{1}
\end{array}\right), \quad[b]=\left(\begin{array}{cc}
0 & \lambda_{4} \\
0 & 0
\end{array}\right),} \\
& {[c]=\left(\begin{array}{cc}
0 & 1 \\
0 & 0
\end{array}\right), \quad[g]=\left(\begin{array}{cc}
\lambda_{2} & 0 \\
0 & \lambda_{2}
\end{array}\right), \quad[h]=\left(\begin{array}{cc}
\lambda_{3} & 0 \\
0 & -\lambda_{3}
\end{array}\right),} \\
& {[x]=\left(\begin{array}{cc}
0 & \frac{\sqrt{2}}{2} \xi \lambda_{1}^{3}\left(\lambda_{2} \lambda_{3}-\lambda_{4}\right) \\
\sqrt{2} \xi \lambda_{1}\left(\lambda_{2} \lambda_{3}+\lambda_{4}\right) & 0
\end{array}\right),}
\end{aligned}
$$

where $\lambda_{1}^{4}=1, \lambda_{2}^{4}=1, \lambda_{3}^{2}=1$ and $\lambda_{4}^{2}=1$. It is clear that $V$ is simple if and only if $\lambda_{2} \lambda_{3}+\lambda_{4} \neq 0$. If $\lambda_{1}=\xi^{i}, \lambda_{2}=\xi^{j}, \lambda_{3}=(-1)^{k}$ and $\lambda_{4}=(-1)^{\iota}$, then $(i, j, k, \iota) \in \Lambda$.

We claim that $V_{i, j, k, \iota} \cong V_{p, q, r, \kappa}$ if and only if $(i, j, k, \iota)=(p, q, r, \kappa)$ in $\Lambda$. Assume that $\Phi: V_{i, j, k, \iota} \mapsto V_{p, q, r, \kappa}$ is an isomorphism of $\mathcal{D}$-modules. Denote by $[\Phi]=$ $\left(p_{i, j}\right)_{i, j=1,2}$ the matrix of $\Phi$ in the given basis. Since $[c][\Psi]=[\Psi][c]$ and $[a][\Psi]=$ $[\Psi][a], p_{21}=0=p_{11}-p_{22}$ and $\left(\xi^{p}-\xi^{i}\right) p_{11}=0=\left(\xi^{p}-\xi^{i+1}\right) p_{12}$. Since $\Psi$ is isomorphic, $\xi^{i}=\xi^{p}$, which implies that $p_{12}=0$ and $[\Phi]=p_{11} I$, where $I$ is the identity matrix. Similarly, $\xi^{j}=\xi^{q}, k=r, \iota=\kappa$. Thus, the claim follows.

Remark 3.6. For a left $\mathcal{D}$-module $V$, there exists a left dual module $V^{*}$ with the module structure given by $(h \rightarrow f)(v)=f(S(h) \cdot v)$ for all $h \in \mathcal{D}, v \in V, f \in V^{*}$. $A$ direct calculation shows that $V_{i, j, k, \iota}^{*} \cong V_{-i-1,-j, k+1, \iota+1}$ for all $(i, j, k, \iota) \in \Lambda$.

Finally, we describe all the simple $\mathcal{D}$-modules up to isomorphism.

Theorem 3.7. There exist 64 simple left $\mathcal{D}$-modules up to isomorphism, among which 16 one-dimensional modules are given in Lemma 3.4 and 48 two-dimensional simple modules are given in Lemma 3.5.

Proof. We first claim that ${ }_{\mathcal{D}} \mathcal{M} \cong{ }_{\mathcal{D}(\operatorname{gr} \mathcal{A})} \mathcal{M}$. Indeed, $\mathcal{D} \mathcal{M} \cong{ }_{H}^{H} \mathcal{Y} \mathcal{D}$ by [25. Proposition 10.6.16] and ${ }_{H}^{H} \mathcal{Y D} \cong{ }_{\mathcal{A}}^{\mathcal{A}} \mathcal{Y D}$ by [5, Proposition 2.2.1]. By [18, Theorem 4.3], $\mathcal{A}$ is a cocycle deformation of gr $A$. Then by [24, Theorem 2.7], ${ }_{\mathcal{A}}^{\mathcal{A}} \mathcal{Y} \mathcal{D} \cong \operatorname{gr}_{\text {gr } \mathcal{A}} \mathcal{Y D}$ and hence the claim follows. Note that gr $\mathcal{A}=\mathcal{B}(W) \sharp \mathbb{k}[\Gamma]$, where $\Gamma \cong \mathbb{Z}_{4} \times \mathbb{Z}_{2}$ with 
generators $g, h$ and $W:=\mathbb{k}\{v\} \in{ }_{\Gamma}^{\Gamma} \mathcal{Y} \mathcal{D}$ with the Yetter-Drinfeld module structure given by $g \cdot x=x, h \cdot x=-x$ and $\delta(x)=g h \otimes x$. A direct computation shows that $\mathcal{D}(\operatorname{gr} \mathcal{A})$ is isomorphic to the Hopf algebra $B$ generated by the elements $g_{1}, g_{2}, g_{3}, g_{4}, x_{1}, x_{2}$ satisfying the relations

$$
\begin{gathered}
g_{i} g_{j}=g_{j} g_{i}, \quad g_{1+k}^{4}=g_{2+k}^{2}=1, \quad x_{k}^{2}=0, \quad x_{1} x_{2}+x_{2} x_{1}=g_{1} g_{2} g_{4}-1, \\
g_{i} x_{1}=\chi\left(g_{i}\right) x_{1} g_{i}, \quad g_{i} x_{2}=\chi^{-1}\left(g_{i}\right) x_{2} g_{i},
\end{gathered}
$$

with the coalgebra structure given by $\Delta\left(g_{i}\right)=g_{i} \otimes g_{i}, \Delta\left(x_{1}\right)=x_{1} \otimes 1+g_{1} g_{2} \otimes x_{1}$ and $\Delta\left(x_{2}\right)=x_{2} \otimes 1+g_{4} \otimes x_{2}$, where $i, j \in \mathbb{I}_{0,3}, k \in \mathbb{I}_{0,1}, \chi\left(g_{1}\right)=1, \chi\left(g_{2}\right)=$ $\chi\left(g_{4}\right)=-1$ and $\chi\left(g_{3}\right)=\xi$. Clearly, $B$ is a lifting of a quantum plane. Thus by [2, Theorem 3.5], $\operatorname{dim} V<3$ for any simple $B$-module $V$. The proposition follows by ${ }_{\mathcal{D}} \mathcal{M} \cong \mathcal{D}(\operatorname{gr} \mathcal{A}) \mathcal{M}$.

3.3. Nichols algebras in ${ }_{H}^{H} \mathcal{Y} \mathcal{D}$. We describe simple objects in ${ }_{H}^{H} \mathcal{Y D}$ and determine all finite-dimensional Nichols algebras over them. We first describe simple objects in ${ }_{H}^{H} \mathcal{Y D}$ by using the equivalence ${ }_{H}^{H} \mathcal{Y D} \cong{ }_{\mathcal{D}} \mathcal{M}$ [25, Proposition 10.6.16].

Lemma 3.8. Let $\mathbb{k}_{\chi_{i, j, k}}=\mathbb{k}\{v\}$ for any $(i, j, k) \in \mathbb{I}_{0,1} \times \mathbb{I}_{0,1} \times \mathbb{I}_{0,3}$. Then $\mathbb{k}_{\chi_{i, j, k}} \in$ ${ }_{H}^{H} \mathcal{Y D}$ with the Yetter-Drinfeld module structure given by

$$
\begin{aligned}
& a \cdot v=\xi^{k} v, \quad b \cdot v=0, \quad c \cdot v=0, \quad d \cdot v=(-1)^{i+j} \xi^{k} v ; \\
& \delta(v)= \begin{cases}a^{2 i} \otimes v & \text { if } j=0 ; \\
d a^{2 i+3} \otimes v & \text { if } j=1 .\end{cases}
\end{aligned}
$$

Proof. Since $\mathbb{k}_{\chi_{i, j, k}}$ is a one-dimensional $\mathcal{D}$-module, the $H$-action is given by the restriction of the character of $\mathcal{D}$ given by Lemma 3.4 and the coaction must be of the form $\delta(v)=t \otimes v$, where $t \in G(H)=\left\{1, a^{2}, d a, d a^{3}\right\}$ such that $\langle g, t\rangle v=(-1)^{i} v$ and $\langle h, t\rangle v=(-1)^{j} v$. Then the lemma follows by Remark $3.2(3)$.

Lemma 3.9. Let $V_{i, j, k, \iota}=\mathbb{k}\left\{v_{1}, v_{2}\right\}$ for $(i, j, k, \iota) \in \Lambda$. Then $V_{i, j, k, \iota} \in{ }_{H}^{H} \mathcal{Y D}$ with the module structure given by

$$
\begin{aligned}
& a \cdot v_{1}=(-1)^{\iota+1} \xi^{i} v_{1}, \quad b \cdot v_{1}=0, \quad c \cdot v_{1}=0, \quad d \cdot v_{1}=\xi^{i} v_{1}, \\
& a \cdot v_{2}=(-1)^{\iota} \xi^{i+1} v_{2}, \quad b \cdot v_{2}=(-1)^{\iota} v_{1}, \quad c \cdot v_{2}=v_{1}, \quad d \cdot v_{2}=\xi^{i+1} v_{2},
\end{aligned}
$$

and the comodule structure given by

(1) if $k=0: \quad \delta\left(v_{1}\right)=a^{j} \otimes v_{1}+w_{2} b a^{j-1} \otimes v_{2}, \quad \delta\left(v_{2}\right)=d a^{j-1} \otimes v_{2}+w_{1} c a^{j-1} \otimes v_{1}$;

(2) if $k=1: \quad \delta\left(v_{1}\right)=d a^{j-1} \otimes v_{1}+w_{2} c a^{j-1} \otimes v_{2}, \delta\left(v_{2}\right)=a^{j} \otimes v_{2}-w_{1} b a^{j-1} \otimes v_{1}$, where $w_{1}=\frac{1}{2} \xi^{3 i+1}\left(\xi^{j}-(-1)^{\iota+k}\right)$ and $w_{2}=\xi^{i+1}\left((-1)^{\iota}+(-1)^{k} \xi^{j}\right)$.

Proof. Note that by Remark 3.2 , we have that

$$
\begin{aligned}
\left(g^{l}\right)^{*}=\frac{1}{8} \sum_{i=0}^{3} \xi^{-i l} a^{i}+\xi^{-(i+1) l} d a^{i}, & \left(g^{l} h\right)^{*}=\frac{1}{8} \sum_{i=0}^{3} \xi^{-i l} a^{i}-\xi^{-(i+1) l} d a^{i}, \\
\left(g^{l} x\right)^{*}=\frac{1}{8 \sqrt{2}} \sum_{i=0}^{3} \xi^{-(i+1) l}\left(b a^{i}+c a^{i}\right), & \left(g^{l} h x\right)^{*}=\frac{1}{8 \sqrt{2}} \sum_{i=0}^{3} \xi^{-(i+1) l}\left(b a^{i}-c a^{i}\right) .
\end{aligned}
$$


Let $\left\{h_{i}\right\}_{1 \leq i \leq 16}$ and $\left\{h^{i}\right\}_{1 \leq i \leq 16}$ be the dual bases of $H$ and $H^{*}$. Then the comodule structure is given by $\delta(v)=\sum_{i=1}^{16} c_{i} \otimes c^{i} \cdot v$ for any $v \in V_{i, j, k, \iota}$. If we denote $\lambda_{1}=\xi^{i}$, $\lambda_{2}=\xi^{j}, \lambda_{3}=(-1)^{k}$ and $\lambda_{4}=(-1)^{\iota}$, then

$$
\begin{aligned}
\delta\left(v_{1}\right)= & \sum_{l=0}^{3} \sum_{n=0}^{1}\left(g^{l} h^{n}\right)^{*} \otimes g^{l} h^{n} \cdot v_{1}+\left(g^{l} h^{n} x\right)^{*} \otimes g^{l} h^{n} x \cdot v_{1} \\
= & \sum_{l=0}^{3} \sum_{n=0}^{1} \lambda_{3}^{n} \lambda_{2}^{l}\left(g^{l} h^{n}\right)^{*} \otimes v_{1}+\lambda_{3}^{n}\left(\lambda_{2}\right)^{l}\left(g^{l} h^{n} x\right)^{*} \otimes x_{2} v_{2} \\
= & \frac{1}{2}\left[\left(1+\lambda_{3}\right) a^{j}+\left(1-\lambda_{3}\right) d a^{j-1}\right] \otimes v_{1}+\frac{1}{2 \sqrt{2}} x_{2}\left(1+\lambda_{3}\right) b a^{j-1} \otimes v_{2} \\
& +\frac{1}{2 \sqrt{2}} x_{2}\left(1-\lambda_{3}\right) c a^{j-1} \otimes v_{2}, \\
\delta\left(v_{2}\right)= & \sum_{l=0}^{3} \sum_{n=0}^{1}\left(\left(g^{l}\right)\left(g^{l} h^{n}\right)^{*} \otimes g^{l} h^{n} \cdot v_{2}+\left(g^{l} h^{n} x\right)^{*} \otimes g^{l} h^{n} x \cdot v_{2}\right. \\
= & \sum_{l=0}^{3} \sum_{n=0}^{1}\left(-\lambda_{3}\right)^{n} \lambda_{2}^{l}\left(g^{l} h^{n}\right)^{*} \otimes v_{2}+\left(-\lambda_{3}\right)^{n}\left(\lambda_{2}\right)^{l}\left(g^{l} h^{n} x\right)^{*} \otimes x_{1} v_{1} \\
= & \frac{1}{2}\left[\left(1-\lambda_{3}\right) a^{j}+\left(1+\lambda_{3}\right) d a^{j-1}\right] \otimes v_{2}+\frac{1}{2 \sqrt{2}} x_{1}\left(1-\lambda_{3}\right) b a^{j-1} \otimes v_{1} \\
& +\frac{1}{2 \sqrt{2}} x_{1}\left(1+\lambda_{3}\right) c a^{j-1} \otimes v_{1},
\end{aligned}
$$

where $x_{1}=\frac{\sqrt{2}}{2} \xi \lambda_{1}^{3}\left(\lambda_{2} \lambda_{3}-\lambda_{4}\right)$ and $x_{2}=\sqrt{2} \xi \lambda_{1}\left(\lambda_{2} \lambda_{3}+\lambda_{4}\right)$.

Remark 3.10. Let $V_{i, j, k, \iota}=\mathbb{k}\left\{v_{1}, v_{2}\right\} \in{ }_{H}^{H} \mathcal{Y D}$ for $(i, j, k, \iota) \in \Lambda$. Then by (2), $V_{i, j, k, \iota} \in{ }_{\mathcal{A}}^{\mathcal{A}} \mathcal{Y} \mathcal{D}$ with the module structure given by

$$
\begin{array}{ll}
g \cdot v_{1}=\xi^{-j} v_{1}, & h \cdot v_{1}=(-1)^{k} v_{1}, \quad x \cdot v_{1}=(-1)^{k+1} x_{2} \xi^{-j} v_{2}, \\
g \cdot v_{2}=\xi^{-j} v_{2}, & h \cdot v_{2}=(-1)^{k+1} v_{2}, \quad x \cdot v_{2}=(-1)^{k} x_{1} \xi^{-j} v_{1},
\end{array}
$$

and the comodule structure given by

(1) for $\iota=0: \quad \delta\left(v_{1}\right)=g^{-2-i} h \otimes v_{1}, \quad \delta\left(v_{2}\right)=g^{-1-i} \otimes v_{2}+\frac{\sqrt{2}}{2} \xi^{1-i} g^{-2-i} h x \otimes v_{1}$,

(2) for $\iota=1: \quad \delta\left(v_{1}\right)=g^{-i} \otimes v_{1}, \quad \delta\left(v_{2}\right)=g^{-i+1} h \otimes v_{2}-\frac{\sqrt{2}}{2} \xi^{1-i} g^{-i} x \otimes v_{1}$,

where $x_{1}=\frac{\sqrt{2}}{2} \xi^{1-i}\left(\xi^{j}(-1)^{k}-(-1)^{\iota}\right)$ and $x_{2}=-\sqrt{2} \xi^{i-1}\left(\xi^{j}(-1)^{k}+(-1)^{\iota}\right)$.

Then we describe the braidings of the simple objects in ${ }_{H}^{H} \mathcal{Y} \mathcal{D}$.

Lemma 3.11. Let $\mathbb{k}_{\chi_{i, j, k}}=\mathbb{k}\{v\} \in{ }_{H}^{H} \mathcal{Y D}$ for $(i, j, k) \in \mathbb{I}_{0,1} \times \mathbb{I}_{0,1} \times \mathbb{I}_{0,3}$. Then the braiding of $\mathrm{k}_{\chi_{i, j, k}}$ is given by

$$
c(v \otimes v)= \begin{cases}(-1)^{i k} v \otimes v, & \text { if } j=0 \\ -(-1)^{(i+1) k} v \otimes v, & \text { if } j=1\end{cases}
$$


Lemma 3.12. Let $V_{i, j, k, \iota}=\mathbb{k}\left\{v_{1}, v_{2}\right\} \in{ }_{H}^{H} \mathcal{Y D}$ for $(i, j, k, \iota) \in \Lambda$. Then the braiding of $V_{i, j, k, \iota}$ is given by:

(1) If $k=0$, then $c\left(\left[\begin{array}{l}v_{1} \\ v_{2}\end{array}\right] \otimes\left[\begin{array}{ll}v_{1} & v_{2}\end{array}\right]\right)=$

$$
\left[\begin{array}{cc}
(-1)^{(\iota+1) j} \xi^{i j} v_{1} \otimes v_{1} & (-1)^{j \iota} \xi^{(i+1) j} v_{2} \otimes v_{1}+c_{12} v_{1} \otimes v_{2} \\
(-1)^{(\iota+1)(j-1)} \xi^{i j} v_{1} \otimes v_{2} & (-1)^{\iota(j-1)} \xi^{(i+1) j} v_{2} \otimes v_{2}+c_{11} v_{1} \otimes v_{1}
\end{array}\right],
$$

where $c_{12}=(-1)^{j(\iota+1)} \xi^{i j}+(-1)^{\iota(j-1)} \xi^{(i+1) j}, c_{11}=\frac{1}{2}(-1)^{\iota(j-1)} \xi^{(j+2) i+j}\left(\xi^{j}-\right.$ $\left.(-1)^{\iota}\right)$.

(2) If $k=1$, then $c\left(\left[\begin{array}{l}v_{1} \\ v_{2}\end{array}\right] \otimes\left[\begin{array}{ll}v_{1} & v_{2}\end{array}\right]\right)=$

$$
\left[\begin{array}{cc}
(-1)^{(\iota+1)(j-1)} \xi^{i j} v_{1} \otimes v_{1} & (-1)^{\iota(j-1)} \xi^{(i+1) j} v_{2} \otimes v_{1}+d_{12} v_{1} \otimes v_{2} \\
(-1)^{(\iota+1) j} \xi^{i j} v_{1} \otimes v_{2} & (-1)^{j \iota} \xi^{(i+1) j} v_{2} \otimes v_{2}-d_{11} v_{1} \otimes v_{1}
\end{array}\right],
$$

where $d_{12}=(-1)^{(\iota+1)(j-1)} \xi^{i j}+(-1)^{j \iota} \xi^{(i+1) j}, d_{11}=\frac{1}{2}(-1)^{j \iota} \xi^{(j+2) i+j}\left(\xi^{j}+(-1)^{\iota}\right)$.

Finally, we determine all finite-dimensional Nichols algebras over simple objects in ${ }_{H}^{H} \mathcal{Y D}$ and present them by generators and relations. We shall show that all finite-dimensional Nichols algebras over the one-dimensional objects in ${ }_{H}^{H} \mathcal{Y} \mathcal{D}$ are parametrized by the set

$$
\Lambda^{0}=\left\{(i, j, k) \in \mathbb{I}_{0,1} \times \mathbb{I}_{0,1} \times \mathbb{I}_{0,3} \mid 2 \nmid i k \text { if } j=0 \text { or, } 2 \mid(i+1) k \text { if } j=1\right\} .
$$

By Lemma 3.11, the next lemma follows immediately.

Lemma 3.13. Let $(i, j, k) \in \mathbb{I}_{0,1} \times \mathbb{I}_{0,1} \times \mathbb{I}_{0,3}$. The Nichols algebra $\mathcal{B}\left(\mathbb{k}_{\chi_{i, j, k}}\right)$ over $\mathbb{k}_{\chi_{i, j, k}}$ is

$$
\mathcal{B}\left(\mathbb{k}_{\chi_{i, j, k}}\right)= \begin{cases}\bigwedge \mathbb{k}_{\chi_{i, j, k}}, & (i, j, k) \in \Lambda^{0} \\ \mathbb{k}[v], & \text { others. }\end{cases}
$$

We shall determine all finite-dimensional Nichols algebras over two-dimensional simple objects in ${ }_{H}^{H} \mathcal{Y} \mathcal{D}$. Set $n \in \mathbb{I}_{0,1}$. Consider the finite subsets of $\Lambda$ given by

$$
\begin{aligned}
\Lambda^{1}= & \{(i, j, k, \iota) \in \Lambda \mid k=0,(2 \iota+2+i) j=0 \bmod 4, \\
& \text { or } k=1,2(\iota+1)(j-1)+i j=0 \bmod 4\}, \\
\Lambda^{1 *}= & \{(i, j, k, \iota) \in \Lambda \mid k=0,2 \iota(j-1)+(i+1) j=0 \bmod 4, \\
& \text { or } k=1,(2 \iota+i+1) j=0 \bmod 4\}, \\
\Lambda^{2}= & \{(i, j, k, \iota) \in \Lambda \mid(i, j, k, \iota) \in\{(2 n, 2,1,0),(2 n+1,2,0,1)\}, \\
\Lambda^{3}= & \{(i, j, k, \iota) \in \Lambda \mid j \in\{1,3\},(i, k, \iota) \in\{(0,0,0),(2,0,1)\} \text { or }(i, k)=(2,1)\}, \\
\Lambda^{4}= & \{(i, j, k, \iota) \in \Lambda \mid j \in\{1,3\},(i, k, \iota) \in\{(1,1,0),(3,1,1)\} \text { or }(i, k)=(1,0)\} .
\end{aligned}
$$

Clearly, $\Lambda=\cup_{i=1}^{4} \Lambda^{i} \cup \Lambda^{1 *}$ and $\left|\Lambda^{2}\right|-4=\left|\Lambda^{3}\right|-8=\left|\Lambda^{4}\right|-8=0$. It turns out that the Nichols algebra $\mathcal{B}\left(V_{i, j, k, \iota}\right)$ is finite-dimensional if $(i, j, k, \iota) \in \cup_{i=2}^{4} \Lambda^{i}$.

Proposition 3.14. Let $V$ be a two-dimensional simple object in ${ }_{H}^{H} \mathcal{Y D}$. Then $\mathcal{B}(V)$ is finite-dimensional if and only if $V$ is isomorphic to $V_{i, j, k, \iota}$ for $(i, j, k, \iota) \in$ $\Lambda^{2} \cup \Lambda^{3} \cup \Lambda^{4}$. Moreover, the generators and relations are given by: 


\begin{tabular}{|c|c|c|}
\hline$(i, j, k, \iota) \in$ & relations of $\mathcal{B}\left(V_{i, j, k, \iota}\right)$ with generators $v_{1}, v_{2}$ & $\operatorname{dim} \mathcal{B}(V)$ \\
\hline$\Lambda^{2}$ & $v_{1}^{2}=0, v_{1} v_{2}-(-1)^{k} v_{2} v_{1}=0, v_{2}^{2}=0$ & 4 \\
\hline$\Lambda^{3}$ & $v_{1}^{2}=0, v_{1} v_{2}-\xi^{(1+2 k) j} v_{2} v_{1}=0, v_{2}^{4}=0$ & 8 \\
\hline$\Lambda^{4}$ & $v_{1}^{4}=0, v_{1} v_{2}+(-1)^{\iota} v_{2} v_{1}=0, v_{1}^{2}+2(-1)^{\iota} v_{2}^{2}=0$ & 8 \\
\hline
\end{tabular}

Proof. We claim that $\operatorname{dim} \mathcal{B}\left(V_{i, j, k, \iota}\right)=\infty$ for $(i, j, k, \iota) \in \Lambda^{1} \cup \Lambda^{1 *}$. Indeed, by Lemma 3.12 , the braiding of $V_{i, j, k, \iota}$ with $(i, j, k, \iota) \in \Lambda^{1}$ has the eigenvector $v_{1} \otimes v_{1}$ of eigenvalue 1. For any $(i, j, k, \iota) \in \Lambda^{1 *}$, there exists $(p, q, r, \mu) \in \Lambda^{1}$ such that $V_{i, j, k, \iota}^{*} \cong V_{p, q, r, \mu}$ in ${ }_{H}^{H} \mathcal{Y} \mathcal{D}$, then by Proposition 2.3 the claim follows.

For $(\alpha, \beta, \mu, \nu) \in \Lambda^{2},(i, j, k, \iota) \in \Lambda^{3} \cup \Lambda^{4}$, a direct computation shows that the braided vector spaces $V_{\alpha, \beta, \mu, \nu}, V_{i, j, k, 1}$ and $V_{i, j, k, 0}$ belong to the cases $\mathfrak{R}_{2,1}, \mathfrak{R}_{1,2}$ and $\mathfrak{R}_{1,2}(a)$ in 22, 4], respectively. That is, the braiding matrices of $V_{\alpha, \beta, \mu, \nu}, V_{i, j, k, 1}$ and $V_{i, j, k, 0}$ are given by

$$
\begin{aligned}
& c\left(\left[\begin{array}{l}
v_{1} \\
v_{2}
\end{array}\right] \otimes\left[\begin{array}{ll}
v_{1} & v_{2}
\end{array}\right]\right)=\left[\begin{array}{cc}
t^{2} v_{1} \otimes v_{1} & t q v_{2} \otimes v_{1}+\left(t^{2}-p q\right) v_{1} \otimes v_{2} \\
t p v_{1} \otimes v_{2} & t^{2} v_{2} \otimes v_{2}
\end{array}\right], \\
& c\left(\left[\begin{array}{l}
v_{1} \\
v_{2}
\end{array}\right] \otimes\left[\begin{array}{ll}
v_{1} & v_{2}
\end{array}\right]\right)=\left[\begin{array}{cc}
p v_{1} \otimes v_{1} & q v_{2} \otimes v_{1}+(p-q) v_{1} \otimes v_{2} \\
p v_{1} \otimes v_{2} & -q v_{2} \otimes v_{2}+k v_{1} \otimes v_{1}
\end{array}\right], \\
& c\left(\left[\begin{array}{l}
v_{1} \\
v_{2}
\end{array}\right] \otimes\left[\begin{array}{ll}
v_{1} & v_{2}
\end{array}\right]\right)=\left[\begin{array}{cc}
-q v_{1} \otimes v_{1} & p v_{2} \otimes v_{1}+(p-q) v_{1} \otimes v_{2} \\
q v_{1} \otimes v_{2} & p v_{2} \otimes v_{2}+k v_{1} \otimes v_{1}
\end{array}\right],
\end{aligned}
$$

respectively. More precisely,

- If $(i, j, k, \iota) \in \Lambda^{2}$, then $t^{2}=-1, t q=(-1)^{k}, t p=-1, t^{2}-p q=-2$;

- if $(i, j, k, 1) \in \Lambda^{3}$, then $p=-1, q=\xi^{(1+2 k) j}, k=\frac{1}{2}\left(1-\xi^{(1+2 k) j}\right)$;

- if $(i, j, k, 0) \in \Lambda^{3}$, then $q=1, p=\xi^{(1+2 k) j}, k=-\frac{1}{2}\left(\xi^{(1+2 k) j}+1\right)$;

- if $(i, j, k, 1) \in \Lambda^{4}$, then $p=\xi^{(1+2 k) j}, q=1, k=\frac{1}{2}\left(1+\xi^{(1+2 k) j}\right)$;

- if $(i, j, k, 0) \in \Lambda^{4}$, then $q=\xi^{(1+2 k) j}, p=-1, k=\frac{1}{2}\left(\xi^{(1+2 k) j}-1\right)$.

Then by [4, Proposition 3.3], 4, Proposition 3.10] and [4, Proposition 3.11], the assertion follows.

Remark 3.15. By Remark 3.6 and Proposition 2.3. $\mathcal{B}\left(V_{0, j, 0,0}\right) \cong \mathcal{B}\left(V_{3,-j, 1,1}\right)^{* b o p}$, $\mathcal{B}\left(V_{2, j, 0,1}\right) \cong \mathcal{B}\left(V_{1,-j, 1,0}\right)^{* \text { bop }}$ and $\mathcal{B}\left(V_{2, j, 1, \iota}\right) \cong \mathcal{B}\left(V_{1,-j, 0, \iota+1}\right)^{* \text { bop }}$, where $j \in\{1,3\}$ and $\iota \in\{0,1\}$. From the proof of Proposition 3.14, the braiding matrices of $V_{2, j_{1}, 0,1}$ and $V_{2, j_{2}, 1,1}$ are, up to a primitive 4 th root of unity, the same and so are $V_{0, j_{1}, 0,0}$ and $V_{2, j_{2}, 1,0}, V_{1, j_{1}, 0,1}$ and $V_{3, j_{2}, 1,1}, V_{1, j_{1}, 0,0}$ and $V_{1, j_{2}, 1,0}$, where $j_{1}, j_{2} \in\{1,3\}$.

Remark 3.16. The Nichols algebras $\mathcal{B}\left(V_{i, j, k, \iota}\right)$ with $(i, j, k, \iota) \in \Lambda^{3}$ and $\mathcal{B}\left(V_{i, j, k, 0}\right)$ with $(i, j, k, 0) \in \Lambda^{4}$ are isomorphic (up to a primitive 4th root of unity) to the Nichols algebras $\mathcal{B}\left(V_{2, j}\right)$ and $\mathcal{B}\left(V_{3, j}\right)$ appearing in [15] as algebras but not as coalgebras since the braidings differ.

Remark 3.17. Let $V_{i, j, k, \iota} \in{ }_{H}^{H} \mathcal{Y D}$ with $(i, j, k, \iota) \in \Lambda^{2} \cup \Lambda^{3} \cup \Lambda^{4}$. Then $V_{i, j, k, \iota} \in$ ${ }_{\mathcal{A}}^{\mathcal{Y}} \mathcal{Y} \mathcal{D}$ with the Yetter-Drinfeld module structure given by Remark 3.10. Denote by $B_{i, j, k, \iota}$ the subalgebra of $\mathcal{B}\left(V_{i, j, k, \iota}\right) \sharp \mathcal{A}$ generated by $g, h, x, v_{1}$. Then $B_{i, j, k, \iota}$ is a pointed Hopf algebra with $\Gamma:=\mathcal{G}\left(B_{i, j, k, \iota}\right) \cong \mathbb{Z}_{4} \times \mathbb{Z}_{2}$. Note that $B_{i, j, k, \iota}$ is isomorphic 
to the quotient of $\mathcal{B}\left(X_{i, j, k, \iota}\right) \sharp \mathbb{k}[\Gamma]$ by the relation $x^{2}=1-g^{2}$, where $X_{i, j, k, \iota}=$ $\mathbb{k}\left\{x, v_{1}\right\} \in{ }_{\Gamma}^{\Gamma} \mathcal{Y} \mathcal{D}$ with the Yetter-Drinfeld module structure given by

$$
\begin{aligned}
& g \cdot x=x, \quad h \cdot x=-x, \quad g \cdot v_{1}=\xi^{-j} v_{1}, \quad h \cdot v_{1}=(-1)^{k} v_{1}, \\
& \delta(x)=g h \otimes x, \quad \delta\left(v_{1}\right)=g^{-2-i} h \otimes v_{1} \text { or } \delta\left(v_{1}\right)=g^{-i} \otimes v_{1}, \quad \text { if } \iota=0 \text { or } \iota=1 .
\end{aligned}
$$

It is easy to see that $\operatorname{gr} B_{i, j, k, \iota} \cong \mathcal{B}\left(X_{i, j, k, \iota}\right) \sharp \mathbb{k}[\Gamma]$ and $\mathcal{B}\left(X_{i, j, k, \iota}\right)$ is of diagonal type with the generalized Dynkin diagram (see [20]) $\begin{gathered}q_{11} \\ 0 \\ x\end{gathered} \underset{v_{1}}{q_{12} q_{21}} \underset{v_{22}}{q_{2}}$ given by

(1) for $\iota=0: q_{11}=-1, q_{12} q_{21}=(-1)^{k+1} \xi^{-j}, q_{22}=(-1)^{k} \xi^{(2+i) j}$;

(2) for $\iota=1: q_{11}=-1, q_{12} q_{21}=(-1)^{k} \xi^{-j}, q_{22}=\xi^{i j}$.

We claim that $\operatorname{dim} B_{i, j, k, \iota}=\operatorname{dim} \mathcal{B}\left(V_{i, j, k, \iota}\right) \sharp \mathcal{A}$ and hence $B_{i, j, k, \iota} \cong \mathcal{B}\left(V_{i, j, k, \iota}\right) \sharp \mathcal{A}$ as Hopf algebras. Indeed, if $(i, j, k, \iota) \in \Lambda^{2}$, then a direct computation shows

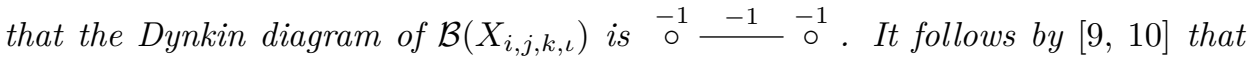
$\operatorname{dim} \mathcal{B}\left(X_{i, j, k, \iota}\right)=8$ and hence

$$
\operatorname{dim} B_{i, j, k, \iota}=\operatorname{dim} \mathcal{B}\left(X_{i, j, k, \iota}\right) \sharp \mathbb{k}[\Gamma]=64=\operatorname{dim} \mathcal{B}\left(V_{i, j, k, \iota}\right) \sharp \mathcal{A} .
$$

If $(i, j, k, \iota) \in \Lambda^{3}$, then the Dynkin diagram is $\stackrel{-1}{\circ} \stackrel{\xi^{ \pm j}}{-1}$ - . If $(i, j, k, \iota) \in \Lambda^{4}$, then the Dynkin diagram is $\begin{gathered}0^{-1} \\ \xi^{ \pm j}\end{gathered} \stackrel{\xi^{\mp j}}{\circ}$. It follows that $\operatorname{dim} \mathcal{B}\left(X_{i, j, k, \iota}\right)=16$ and hence $\operatorname{dim} B_{i, j, k, \iota}=128=\operatorname{dim} \mathcal{B}\left(V_{i, j, k, \iota}\right) \sharp \mathcal{A}$.

We claim that gr $B_{i, j, k, \iota} \cong \mathcal{B}\left(V_{i, j, k, \iota}\right) \sharp$ gr $\mathcal{A}$. Recall that gr $\mathcal{A} \cong \mathcal{A}_{\sigma}$ for some Hopf 2-cocycle $\sigma$. By [19, Proposition 4.2], $\sigma=\epsilon \otimes \epsilon-\zeta$, where $\zeta\left(x^{i} g^{j} h^{k}, x^{m} g^{n} h^{l}\right)=$ $(-1)^{m k} \delta_{2, i+m}$ for $i, k, m, l \in \mathbb{I}_{0,1}, j, n \in \mathbb{I}_{0,3}$. By [24, Theorem 2.7], a direct com-

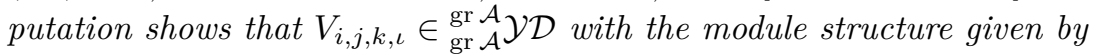

$$
\begin{array}{ll}
g \cdot v_{1}=\xi^{-j} v_{1}, & h \cdot v_{1}=(-1)^{k} v_{1}, \quad x \cdot v_{1}=\alpha_{1} v_{1}+\alpha_{2} v_{2}, \\
g \cdot v_{2}=\xi^{-j} v_{2}, & h \cdot v_{2}=(-1)^{k+1} v_{2}, \quad x \cdot v_{2}=\beta_{1} v_{1}+\beta_{2} v_{2},
\end{array}
$$

and the comodule structure given by

(1) for $\iota=0: \delta\left(v_{1}\right)=g^{-2-i} h \otimes v_{1}, \delta\left(v_{2}\right)=g^{-1-i} \otimes v_{2}+\frac{\sqrt{2}}{2} \xi^{1-i} g^{-2-i} h x \otimes v_{1}$;

(2) for $\iota=1: \delta\left(v_{1}\right)=g^{-i} \otimes v_{1}, \delta\left(v_{2}\right)=g^{-i+1} h \otimes v_{2}-\frac{\sqrt{2}}{2} \xi^{1-i} g^{-i} x \otimes v_{1}$, where $\alpha_{1}, \alpha_{2}, \beta_{1}, \beta_{2} \in \mathbb{k}$. By [21, Proposition 8.8], $\mathcal{B}\left(V_{i, j, k, \iota}\right) \sharp \mathcal{B}(W) \cong \mathcal{B}\left(X_{i, j, k, \iota}\right)$ and hence the claim follows.

From the preceding discussion, the Nichols algebras of dimension greater than 2 in Proposition 3.14 can be related to the Nichols algebras $\mathcal{B}\left(X_{i, j, k, \iota}\right)$ of diagonal type. More precisely, if $(i, j, k, \iota) \in \Lambda^{2}$, then the Dynkin diagram of $\mathcal{B}\left(X_{i, j, k, \iota}\right)$ is ${ }^{-1}-1{ }^{-1}$. If $(i, j, k, \iota) \in \Lambda^{3}$, then the Dynkin diagram is $0^{-1} \xi^{ \pm j}-1$. If $(i, j, k, \iota) \in \Lambda^{4}$, then the Dynkin diagram is $\stackrel{-1}{\circ} \xi^{ \pm j}{\stackrel{\xi^{\mp j}}{\circ}}^{\circ}$. These generalized Dynkin diagrams appeared in the second row or the third row in [20, Table 1]. They

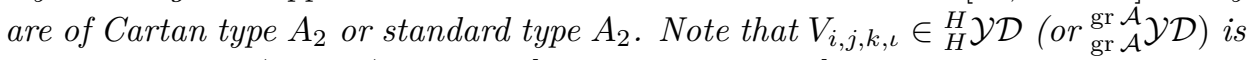
characterized by $(i, j, k, \iota) \in \Lambda$. By [21, Proposition 8.6], we are able to obtain these 
Nichols algebras $\mathcal{B}\left(V_{i, j, k, \iota}\right)$ (up to isomorphism) by splitting the Nichols algebras $\mathcal{B}\left(X_{i, j, k, \iota}\right)$ of diagonal type.

It should be mentioned that a similar idea was used in a recent work [1] to study the Nichols algebras over basic Hopf algebras. In particular, our examples can be recovered in a similar way.

3.4. Hopf algebras over $H$. We determine all finite-dimensional Hopf algebras over $H$ such that their diagrams are strictly graded and their infinitesimal braidings are simple objects in ${ }_{H}^{H} \mathcal{Y} \mathcal{D}$. We first show that the diagrams of these Hopf algebras are Nichols algebras.

Lemma 3.18. Let $A$ be a finite-dimensional Hopf algebra over $H$ such that the corresponding infinitesimal braiding $V$ is a simple object in ${ }_{H}^{H} \mathcal{Y} \mathcal{D}$. Assume that the diagram of $A$ is strictly graded. Then gr $A \cong \mathcal{B}(V) \# H$.

Proof. Let $S$ be the graded dual of the diagram $R$ of $A$. Then by the duality principle [8, Lemma 2.4], $S$ is generated by $S(1)$ if and only if $\mathcal{P}(R)=R(1)$. Since $R$ is strictly graded, there exists an epimorphism $S \rightarrow \mathcal{B}(W)$, where $W:=S(1)$. If $V$ is a simple object in ${ }_{H}^{H} \mathcal{Y D}$, then by Remark 3.6 . $W$ must be simple in ${ }_{H}^{H} \mathcal{Y D}$. To show that $R$ is generated by $R(1)$, it suffices to show that $S$ is a Nichols algebra, that is, to show that the relations of $\mathcal{B}(W)$ also hold in $S$.

Assume $W=\mathbb{k}_{\chi_{i, j, k}}:=\mathbb{k}\{v\}$ with $(i, j, k) \in \Lambda^{0}$. Then $\mathcal{B}(W)=\bigwedge \mathbb{k}_{\chi_{i, j, k}}$ for $(i, j, k) \in \Lambda^{0}$. Suppose that $v^{2} \neq 0$ in $S$. Since $c(v \otimes v)=-v \otimes v$, it follows that $v^{2} \in \mathcal{P}(S)$ and $c\left(v^{2} \otimes v^{2}\right)=v^{2} \otimes v^{2}$, which implies that $\operatorname{dim} S=\infty$, a contradiction. Therefore, the relation $v^{2}=0$ holds in $S$.

Assume that $W=V_{i, j, 0, \iota}$ with $(i, j, 0, \iota) \in \Lambda^{3}$. Set $r_{1}=v_{1} v_{2}-\xi^{j} v_{2} v_{1}$ for simplicity. By Proposition $3.14 \mathcal{B}(W):=\mathbb{k}\left\langle v_{1}, v_{2} \mid v_{1}^{2}=0, r_{1}=0, v_{2}^{4}=0\right\rangle$ and the relations of $\mathcal{B}(W)$ are all primitive elements. As $\delta\left(v_{1}\right)=a^{j} \otimes v_{1}+\xi^{i+1}\left((-1)^{\iota}+\right.$ $\left.\xi^{j}\right) b a^{j-1} \otimes v_{2}$ and $\delta\left(v_{2}\right)=d a^{j-1} \otimes v_{2}+\frac{1}{2} \xi^{3 i+1}\left(\xi^{j}-(-1)^{\iota}\right) c a^{j-1} \otimes v_{1}$, we have that

$$
\delta\left(v_{1}^{2}\right)=a^{2} \otimes v_{1}^{2}+\xi^{i+j+1}\left((-1)^{\iota}+\xi^{j}\right) b a \otimes r_{1}, \quad \delta\left(r_{1}\right)=d a \otimes r_{1} .
$$

Then by the formula defining the braiding in ${ }_{H}^{H} \mathcal{Y} \mathcal{D}, c(r \otimes r)=r \otimes r$ for $r=v_{1}^{2}, r_{1}$ and hence $v_{1}^{2}=0=r_{1}$ in $S$. Finally, we have that $\delta\left(v_{2}^{4}\right)=1 \otimes v_{2}^{4}$, which implies that $c\left(v_{2}^{4} \otimes v_{2}^{4}\right)=v_{2}^{4} \otimes v_{2}^{4}$ and hence $v_{2}^{4}=0$ in $S$.

Similarly, the claim follows for the remaining cases.

Next, we shall show that there do not exist non-trivial liftings for the bosonizations of the Nichols algebras over $\mathbb{k}_{\chi_{i, j, k}}$ with $(i, j, k) \in \Lambda^{0}$ and over $V_{i, j, k, \iota}$ with $(i, j, k, \iota) \in \Lambda^{2} \cup \Lambda^{3}$.

Proposition 3.19. Let $A$ be a finite-dimensional Hopf algebra over $H$ such that gr $A \cong \mathcal{B}(V) \sharp H$, where $V$ is isomorphic either to $\mathbb{k}_{\chi_{i, j, k}}$ for $(i, j, k) \in \Lambda^{0}$ or to $V_{i, j, k, \iota}$ for $(i, j, k, \iota) \in \Lambda^{2} \cup \Lambda^{3}$. Then $A \cong \operatorname{gr} A$.

Proof. We prove the assertion for $V \cong V_{i, j, k, \iota}$ with $(i, j, k, \iota) \in \Lambda^{3}$, being the proof for $\mathrm{k}_{\chi_{i, j, k}}$ and $(i, j, k, \iota) \in \Lambda^{2}$ completely analogous. Note that gr $A \cong \mathcal{B}\left(V_{i, j, k, \iota}\right) \sharp H$ for $(i, j, k, \iota) \in \Lambda^{3}$. We prove the assertion by showing that the relations of $\operatorname{gr} A$ also hold in $A$. 
Assume that $k=0$. Then $\mathcal{B}\left(V_{i, j, 0, \iota}\right) \sharp H$ is generated by $x, y, a, b, c, d$, subject to the relations of $H$, the relations of $\mathcal{B}\left(V_{i, j, 1, \iota}\right)$ and the relations

$$
\begin{gathered}
a x=-x a, \quad b x=-x b, \quad c x=\xi^{i} x c, \quad d x=\xi^{i} x d, \quad a y-\xi y a=(-1)^{\iota} x c, \\
b y-\xi y b=(-1)^{\iota} x d, \quad c y-\xi^{i+1} y c=x a, \quad d y-\xi^{i+1} y d=x b .
\end{gathered}
$$

The coalgebra structure is given by (6), (7), $\Delta(x)=x \otimes 1+a^{j} \otimes x+\xi\left(1+\xi^{i+j}\right) b a^{j-1} \otimes$ $y$ and $\Delta(y)=y \otimes 1+d a^{j-1} \otimes y+\frac{1}{2} \xi^{3 i+1}\left(\xi^{j}-(-1)^{\iota}\right) c a^{j-1} \otimes x$. Then

$$
\begin{gathered}
\Delta\left(x^{2}\right)=x^{2} \otimes 1+a^{2} \otimes x^{2}+\xi\left(\xi^{j}-\xi^{i}\right) b a \otimes\left(x y-\xi^{j} y x\right), \\
\Delta\left(x y-\xi^{j} y x\right)=\left(x y-\xi^{j} y x\right) \otimes 1+d a \otimes\left(x y-\xi^{j} y x\right) .
\end{gathered}
$$

From the second equation, we have $x y-\xi^{j} y x \in \mathcal{P}_{1, d a}(\mathcal{B}(W) \sharp H)=\mathcal{P}_{1, d a}(H)$. Since $\mathcal{P}_{1, d a}(H)=\mathbb{k}\{1-d a\}$, it follows that $x y-\xi^{j} y x=\mu(1-d a)$ for some $\mu \in \mathbb{k}$. Then from the first equation, we get that

$$
\Delta\left(x^{2}+\xi\left(\xi^{j}-\xi^{i}\right) \mu b a\right)=\left(x^{2}+\xi\left(\xi^{j}-\xi^{i}\right) \mu b a\right) \otimes 1+a^{2} \otimes\left(x^{2}+\xi\left(\xi^{j}-\xi^{i}\right) \mu b a\right),
$$

which implies that $x^{2}+\xi\left(\xi^{j}-\xi^{i}\right) \mu b a=\nu\left(1-a^{2}\right)$ for some $\nu \in \mathbb{k}$. Since $a x^{2}=x^{2} a$, $b x^{2}=x^{2} b$ and $a b=\xi b a$, it follows that $\mu=0=\nu$ and hence $x^{2}=0=x y-\xi^{j} y x$ in $A$. Finally, a tedious computation shows that $y^{4} \in \mathcal{P}(A)$ and hence the relation $y^{4}=0$ holds in $A$. Consequently, $A \cong \operatorname{gr} A$.

Assume that $k=1$. Then $\mathcal{B}\left(V_{i, j, 1, \iota}\right) \sharp H$ is generated by $x, y, a, b, c, d$, subject to the relations of $H$, the relations of $\mathcal{B}\left(V_{i, j, 1, \iota}\right)$ and the relations

$$
\begin{gathered}
b x=(-1)^{\iota} x b, \quad c x=-x c, \quad d x=-x d, \quad a y+\xi(-1)^{\iota} y a=(-1)^{\iota} x c, \\
a x=(-1)^{\iota} x a, \quad b y+\xi(-1)^{\iota} y b=(-1)^{\iota} x d, \quad c y+\xi y c=x a, \quad d y+\xi y d=x b .
\end{gathered}
$$

The coalgebra structure is given by (6), (7), $\Delta(x)=x \otimes 1+d a^{j-1} \otimes x+\xi\left(\xi^{j}-\right.$ $\left.(-1)^{\iota}\right) c a^{j-1} \otimes y$ and $\Delta(y)=y \otimes 1+a^{j} \otimes y+\frac{1}{2} \xi\left(\xi^{j}+(-1)^{\iota}\right) b a^{j-1} \otimes x$.

A direct computation shows that $x y+\xi^{j} y x \in \mathcal{P}_{1, d a}\left(\mathcal{B}\left(V_{i, j, 1, \iota}\right) \sharp H\right)=\mathcal{P}_{1, d a}(H)$. Since $\mathcal{P}_{1, d a}(H)=\mathbb{k}\{1-d a\}, x y+\xi^{j} y x=\mu(1-d a)$ for some $\mu \in \mathbb{k}$. Then it follows by a direct computation that $x^{2}+\xi\left(1+\xi^{j}(-1)^{\iota}\right) \mu b a \in \mathcal{P}_{1, a^{2}}\left(\mathcal{B}\left(V_{i, j, 1, \iota}\right) \sharp H\right)=$ $\mathcal{P}_{1, a^{2}}(H)$. Since $\mathcal{P}_{1, a^{2}}(H)=\mathbb{k}\left\{1-a^{2}\right\}, x^{2}+\xi\left(1+\xi^{j}(-1)^{\iota}\right) \mu b a=\nu\left(1-a^{2}\right)$ for some $\nu \in \mathbb{k}$. Since $a x^{2}=x^{2} a, b x^{2}=x^{2} b$ and $a b=\xi b a$, it follows that $\mu=0=\nu$ and hence $x^{2}=0=x y+\xi^{j} y x$ in $A$. Finally, $\Delta\left(y^{4}\right)=\Delta(y)^{4}=y^{4} \otimes 1+1 \otimes y^{4}$, which implies that the relation $y^{4}=0$ holds in $A$. Consequently, $A \cong \operatorname{gr} A$.

Now we define eight families of Hopf algebras $\mathfrak{C}_{i, j, k, \iota}^{4}(\mu)$ depending on the parameter $\mu \in \mathbb{k}$ and show that they are indeed liftings of the Nichols algebras $\mathcal{B}\left(V_{i, j, k, \iota}\right)$ for $(i, j, k, \iota) \in \Lambda^{4}$.

Definition 3.20. For $\mu \in \mathbb{k}$ and $(i, j, 0, \iota) \in \Lambda^{4}$, let $\mathfrak{C}_{i, j, 0, \iota}^{4}(\mu)$ be the algebra generated by $x, y, a, b, c, d$ subject to the relations (4), (5) and

$$
\begin{gathered}
a x=-(-1)^{\iota} \xi x a, \quad b x=-(-1)^{\iota} \xi x b, \quad c x=\xi x c, \quad d x=\xi x d, \\
a y+(-1)^{\iota} y a=(-1)^{\iota} x c, \quad b y+(-1)^{\iota} y b=(-1)^{\iota} x d, c y+y c=x a, d y+y d=x b, \\
x^{2}+2(-1)^{\iota} y^{2}=\mu\left(1-a^{2}\right), \quad x y+(-1)^{\iota} y x=\mu c a, \quad x^{4}=0 .
\end{gathered}
$$


$\mathfrak{C}_{i, j, 0, \iota}^{4}(\mu)$ is a Hopf algebra whose coalgebra structure is given by (6), 7) and

$$
\begin{aligned}
& \Delta(x)=x \otimes 1+a^{j} \otimes x-\left(\xi^{j}+(-1)^{\iota}\right) b a^{j-1} \otimes y, \\
& \Delta(y)=y \otimes 1+d a^{j-1} \otimes y+\frac{1}{2}\left(\xi^{j}-(-1)^{\iota}\right) c a^{j-1} \otimes x .
\end{aligned}
$$

Remark 3.21. It is clear that $\mathfrak{C}_{i, j, 0, \iota}^{4}(0) \cong \mathcal{B}\left(V_{i, j, 0, \iota}\right) \sharp H$ and $\mathfrak{C}_{i, j, 0, \iota}^{4}(\mu)$ with $\mu \neq 0$ is not isomorphic to $\mathfrak{C}_{i, j, 0, \iota}^{4}(0)$ as Hopf algebras for $(i, j, 0, \iota) \in \Lambda^{4}$. Moreover, $\mathfrak{C}_{i, j, 0, \iota}^{4}(\mu) \cong T\left(V_{i, j, k, \iota}\right) \sharp H / J^{0}$, where $J^{0}$ is the ideal generated by the elements given by the last row of the equations in Definition 3.20.

Definition 3.22. For $\mu \in \mathbb{k}$ and $(i, j, 1, \iota) \in \Lambda^{4}$, let $\mathfrak{C}_{i, j, 1, \iota}^{4}(\mu)$ be the algebra generated by $x, y, a, b, c, d$ subject to the relations (4), (5) and

$$
\begin{gathered}
a x=-(-1)^{\iota} \xi^{i} x a, \quad b x=-(-1)^{\iota} \xi^{i} x b, \quad c x=\xi^{i} x c, \quad d x=\xi^{i} x d, \\
a y+y a=(-1)^{\iota} x c, \quad b y+y b=(-1)^{\iota} x d, \quad c y-\xi^{i+1} y c=x a, \quad d y-\xi^{i+1} y d=x b, \\
x^{2}+2(-1)^{\iota} y^{2}=\mu\left(1-a^{2}\right), \quad x y+(-1)^{\iota} y x=(-1)^{\iota} \mu c a, \quad x^{4}=0 .
\end{gathered}
$$

$\mathfrak{C}_{i, j, 1, L}^{4}(\mu)$ is a Hopf algebra whose coalgebra structure is given by (6), (7) and

$$
\begin{aligned}
& \Delta(x)=x \otimes 1+d a^{j-1} \otimes x+\left((-1)^{\iota} \xi^{j}-1\right) c a^{j-1} \otimes y, \\
& \Delta(y)=y \otimes 1+a^{j} \otimes y-\frac{1}{2}\left((-1)^{\iota} \xi^{j}+1\right) b a^{j-1} \otimes x .
\end{aligned}
$$

Remark 3.23. It is clear that $\mathfrak{C}_{i, j, 1, \iota}^{4}(0) \cong \mathcal{B}\left(V_{i, j, 1, \iota}\right) \sharp H$ and $\mathfrak{C}_{i, j, 1, \iota}^{4}(\mu)$ with $\mu \neq 0$ is not isomorphic to $\mathfrak{C}_{i, j, 1, \iota}^{4}(0)$ as Hopf algebras for $(i, j, 1, \iota) \in \Lambda^{4}$. Moreover, $\mathfrak{C}_{i, j, 1, \iota}^{4}(\mu) \cong T\left(V_{i, j, k, \iota}\right) \sharp H / J^{1}$, where $J^{1}$ is the ideal generated by the elements given by the last row of the equations in Definition 3.22.

Lemma 3.24. A linear basis of $\mathfrak{C}_{i, j, k, \iota}^{4}(\mu)$ is given by

$$
\left\{y^{r} x^{s} d^{t} c^{u} b^{v} a^{w}, s, w \in \mathbb{I}_{0,3}, r, t+u+v \in \mathbb{I}_{0,1}\right\} .
$$

In particular, $\operatorname{dim} \mathfrak{C}_{i, j, k, \iota}^{4}(\mu)=128$.

Proof. We prove the assertion for $\mathfrak{C}_{i, j, k, \iota}^{4}(\mu)$ by applying the Diamond Lemma [13] with the order $y<x<d<c<b<a$. By the Diamond Lemma, it suffices to show that all overlap ambiguities are resolvable, that is, the ambiguities can be reduced to the same expression by different substitution rules. To verify all the ambiguities are resolvable is tedious but straightforward. Here we only check the overlaps $(x y) y=x\left(y^{2}\right), x^{3}(x y)=\left(x^{4}\right) y$ and $(a y) y=a\left(y^{2}\right)$.

Assume $k=0$. Note that $a x^{2}=-x^{2} a$. After a direct computation, $c a y=$ $(-1)^{\iota} y c a-(-1)^{\iota} x a^{2}$. Then

$$
\begin{aligned}
(x y) y & =-(-1)^{\iota} y x y+\mu c a y=-(-1)^{\iota} y\left(\mu c a-(-1)^{\iota} y x\right)+\mu c a y \\
& =-(-1)^{\iota} \mu y c a+y^{2} x+\mu c a y=y^{2} x+\mu\left(c a y-(-1)^{\iota} y c a\right) \\
& =y^{2} x-(-1)^{\iota} \mu x a^{2}=\frac{1}{2}(-1)^{\iota}\left[\mu\left(1-a^{2}\right)-x^{2}\right] x-(-1)^{\iota} \mu x a^{2} \\
& =\frac{1}{2}(-1)^{\iota} \mu x\left(1-a^{2}\right)-\frac{1}{2}(-1)^{\iota} x^{3}=x\left(y^{2}\right) .
\end{aligned}
$$


Note that $c a x=(-1)^{\iota} x c a, x(x y)=-(-1)^{\iota} x y x+\mu x c a=-(-1)^{\iota}\left(-(-1)^{\iota} y x+\right.$ $\mu c a) x+\mu x c a=y x^{2}-(-1)^{\iota} \mu c a x+\mu x c a=y x^{2}$. It follows that $x^{3}(x y)=y x^{4}=$ $0=\left(x^{4}\right) y$. Similarly, we have that

$$
\begin{aligned}
(a y) y & =-(-1)^{\iota} y a y+(-1)^{\iota} x c y=-y(-y a+x c)+(-1)^{\iota} x(x a-y c) \\
& =y^{2} a+(-1)^{\iota} x^{2} a-\left(y x+(-1)^{\iota} x y\right) c=y^{2} a+(-1)^{\iota} x^{2} a-\mu(-1)^{\iota} c a c \\
& =\frac{1}{2}(-1)^{\iota} \mu\left(1-a^{2}\right) a+\frac{1}{2}(-1)^{\iota} x^{2} a=\frac{1}{2}(-1)^{\iota} \mu\left(a-a^{3}\right)-\frac{1}{2}(-1)^{\iota} a x^{2}=a\left(y^{2}\right) .
\end{aligned}
$$

Assume $k=1$. The proof follows the same line as for $k=0$.

Now we show that $\mathfrak{C}_{i, j, k, \iota}^{4}(\mu)$ is a lifting of the bosonization $\mathcal{B}\left(V_{i, j, k, \iota}\right) \sharp H$ for $(i, j, k, \iota) \in \Lambda^{4}$.

Lemma 3.25. For $(i, j, k, \iota) \in \Lambda^{4}$, gr $\mathfrak{C}_{i, j, k, \iota}^{4}(\mu) \cong \mathcal{B}\left(V_{i, j, k, \iota}\right) \sharp H$.

Proof. Let $\Lambda_{0}$ be the subalgebra of $\mathfrak{C}_{i, j, k, \iota}^{4}(\mu)$ generated by the subcoalgebra $C=$ $\mathbb{k}\{a, b, c, d\}$. We claim that $\Lambda_{0} \cong H$. Indeed, consider the Hopf algebra map $\psi: H \mapsto \mathfrak{C}_{i, j, k, \iota}^{4}(\mu)$ given by the composition $H \hookrightarrow T\left(V_{i, j, k, \iota}\right) \sharp H \rightarrow \mathfrak{C}_{i, j, k, \iota}^{4}(\mu) \cong$ $T\left(V_{i, j, k, \iota}\right) \sharp H / J^{k}$. It is clear that $\psi(C) \cong C$ as coalgebras and $\psi(H) \cong \Lambda_{0}$ as Hopf algebras. By Lemma $3.24 \operatorname{dim} \Lambda_{0}=16$. Hence $\operatorname{dim} \psi(H)=16$ and $\psi(H) \cong H$, which implies that the claim follows.

Let $\Lambda_{1}=\Lambda_{0}+H\{x, y\}, \Lambda_{2}=\Lambda_{1}+H\left\{x^{2}, x y\right\}, \Lambda_{3}=\Lambda_{2}+H\left\{x^{3}, x^{2} y\right\}$ and $\Lambda_{4}=$ $\Lambda_{3}+H\left\{x^{3} y\right\}$. A direct computation shows that $\left\{\Lambda_{\ell}\right\}_{\ell=0}^{4}$ is a coalgebra filtration of $\mathfrak{C}_{i, j, k, \iota}^{4}(\mu)$. Hence, $\left(\mathfrak{C}_{i, j, k, \iota}^{4}(\mu)\right)_{0} \subseteq H$, which implies that $\left(\mathfrak{C}_{i, j, k, \iota}^{4}(\mu)\right)_{[0]} \cong H$. Therefore, gr $\mathfrak{C}_{i, j, k, \iota}^{4}(\mu) \cong R_{i, j, k, \iota}^{4} \sharp H$. By definition, it is easy to see that $V_{i, j, k, \iota} \subset$ $\mathcal{P}\left(R_{i, j, k, \iota}^{4}\right)$. Then by Lemma $3.24, \operatorname{dim} R_{i, j, k, \iota}^{4}=8=\operatorname{dim} \mathcal{B}\left(V_{i, j, k, \iota}\right)$. Consequently, $\operatorname{gr} \mathfrak{C}_{i, j, k, \iota}^{4}(\mu) \cong \mathcal{B}\left(V_{i, j, k, \iota}\right) \sharp H$.

Proposition 3.26. Let $A$ be a finite-dimensional Hopf algebra over $H$ such that $\operatorname{gr} A \cong \mathcal{B}(V) \sharp H$, where $V$ is isomorphic to $V_{i, j, k, \iota}$, for $(i, j, k, \iota) \in \Lambda^{4}$. Then $A \cong$ $\mathfrak{C}_{i, j, k, \iota}^{4}(\mu)$.

Proof. Assume $k=0$. Then gr $A \cong \mathfrak{C}_{i, j, 0, \iota}^{4}(0)$ as Hopf algebras. As $\Delta(x)=x \otimes 1+$ $a^{j} \otimes x-\left(\xi^{j}+(-1)^{\iota}\right) b a^{j-1} \otimes y$ and $\Delta(y)=y \otimes 1+d a^{j-1} \otimes y+\frac{1}{2}\left(\xi^{j}-(-1)^{\iota}\right) c a^{j-1} \otimes x$,

$$
\begin{aligned}
\Delta\left(x^{2}+2(-1)^{\iota} y^{2}\right)= & \left(x^{2}+2(-1)^{\iota} y^{2}\right) \otimes 1+a^{2} \otimes\left(x^{2}+2(-1)^{\iota} y^{2}\right), \\
\Delta\left(x y+(-1)^{\iota} y x\right)= & \left(x y+(-1)^{\iota} y x\right) \otimes 1-c a \otimes\left(x^{2}+2(-1)^{\iota} y^{2}\right) \\
& +d a \otimes\left(x y+(-1)^{\iota} y x\right) .
\end{aligned}
$$

From the first equation, we have that $x^{2}+2(-1)^{\iota} y^{2} \in \mathcal{P}_{1, a^{2}}\left(\mathcal{B}\left(V_{i, j, 0, \iota}\right) \sharp H\right)=$ $\mathcal{P}_{1, a^{2}}(H)$. Since $\mathcal{P}_{1, a^{2}}(H)=\mathbb{k}\left\{1-a^{2}\right\}$, it follows that $x^{2}+2(-1)^{\iota} y^{2}=\mu\left(1-a^{2}\right)$ for some $\mu \in \mathbb{k}$. Then from the second equation, we get that

$\Delta\left(x y+(-1)^{\iota} y x-\mu c a\right)=\left(x y+(-1)^{\iota} y x-\mu c a\right) \otimes 1+d a \otimes\left(x y+(-1)^{\iota} y x-\mu c a\right)$.

Thus $x y+(-1)^{\iota} y x-\mu c a=\nu(1-d a)$ for some $\nu \in \mathbb{k}$. Since $\nu(1-d a) c=$ $\nu c(1-d a)$ and $c\left(x y+(-1)^{\iota} y x\right)=-\xi\left(x y+(-1)^{\iota} y x\right) c$, it follows that $\nu=0$ and 
hence $x y+(-1)^{\iota} y x=\mu c a$. Finally, $\Delta\left(x^{4}\right)=\Delta(x)^{4}=x^{4} \otimes 1+1 \otimes x^{4}$ and hence the relation $x^{4}=0$ must hold in $A$.

Since the defining relations of $\mathfrak{C}_{i, j, 0, \iota}^{4}(\mu)$ hold in $A$, there is a Hopf algebra epimorphism from $\mathfrak{C}_{i, j, 0, \iota}^{4}(\mu)$ to $A$. Since $\operatorname{dim} A=\operatorname{dim} \mathfrak{C}_{i, j, 0, \iota}^{4}(\mu)$ by Lemma 3.24 it follows that $A \cong \mathfrak{C}_{i, j, 0, \iota}^{4}(\mu)$.

Assume $k=1$. Then gr $A \cong \mathfrak{C}_{i, j, 1, \iota}^{4}(0)$ as Hopf algebras. As $\Delta(x)=x \otimes 1+$ $d a^{j-1} \otimes x+\left((-1)^{\iota} \xi^{j}-1\right) c a^{j-1} \otimes y$ and $\Delta(y)=y \otimes 1+a^{j} \otimes y-\frac{1}{2}\left((-1)^{\iota} \xi^{j}+1\right) b a^{j-1} \otimes x$, a direct computation shows that $x^{2}+2(-1)^{\iota} y^{2}=\mu\left(1-a^{2}\right)$ and $x y+(-1)^{\iota} y x-$ $(-1)^{\iota} \mu c a=\nu(1-d a)$ for some $\mu, \nu \in \mathbb{k}$. Since $c\left(x y+(-1)^{\iota} y x\right)=-\xi\left(x y+(-1)^{\iota} y x\right) c$ and $c(1-d a)=(1-d a) c$, it follows that $\nu=0$ and $x y+(-1)^{\iota} y x=(-1)^{\iota} \mu c a$. Finally, $\Delta\left(x^{4}\right)=\Delta(x)^{4}=x^{4} \otimes 1+1 \otimes x^{4}$ and hence $x^{4}=0$ in $A$. Since the defining relations of $\mathfrak{C}_{i, j, 1, \iota}^{4}(\mu)$ hold in $A$, there is a Hopf algebra epimorphism from $\mathfrak{C}_{i, j, 1, \iota}^{4}(\mu)$ to $A$. By Lemma $3.24 \operatorname{dim} A=\operatorname{dim} \mathfrak{C}_{i, j, 1, \iota}^{4}(\mu)$ and hence $A \cong \mathfrak{C}_{i, j, 1, \iota}^{4}(\mu)$.

Finally, we have the classification of finite-dimensional Hopf algebras over $H$ such that their diagrams are strictly graded and their infinitesimal braidings are simple objects in ${ }_{H}^{H} \mathcal{Y} \mathcal{D}$.

Proof of Theorem A. The Hopf algebras from different families are pairwise non-isomorphic since their infinitesimal braidings are pairwise non-isomorphic as Yetter-Drinfeld modules over $H$. And the rest of assertions follow by Lemmas 3.13 and 3.18 , and Propositions $3.14,3.19$ and 3.26

\section{On Finite-dimensional Hopf algebras over $\widetilde{H}$}

In this section, we determine all finite-dimensional Nichols algebras over simple objects in $\underset{\widetilde{H}}{\widetilde{H}} \mathcal{Y} \mathcal{D}$ and their liftings. These Nichols algebras have already appeared in [15, 4] and consist of 2-dimensional exterior algebras, 4- and 8-dimensional algebras with non-diagonal braidings. The bosonizations of these Nichols algebras are finitedimensional Hopf algebras over $\widetilde{H}$ without the dual Chevalley property. Moreover, the non-trivial liftings of these Nichols algebras might constitute new examples of Hopf algebras of dimension 128 without the dual Chevalley property.

4.1. Finite-dimensional Nichols algebras in $\underset{H}{\widetilde{H}} \mathcal{Y} \mathcal{D}$. We firstly describe the Hopf algebra $\widetilde{H}$, which already appeard in [11, 16] and is generated by its coradical as follows:

Definition 4.1. $\widetilde{H}$ as an algebra is generated by $a, b, c$ satisfying the relations

$$
a^{4}=1, \quad b^{2}=1, \quad c^{2}=0, \quad a c=\xi c a, \quad b a=a b, \quad b c=c b,
$$

and as a coalgebra is given by

$$
\Delta(a)=a \otimes a+a^{2} c \otimes c, \quad \Delta(b)=b \otimes b, \quad \Delta(c)=c \otimes a+a^{3} \otimes c,
$$

and its antipode is given by $S(a)=a^{3}, S(b)=b, S(c)=\xi^{3} c$.

Remark 4.2.

(1) $\mathcal{G}(\widetilde{H})=\left\{1, a^{2}, b, a^{2} b\right\}, \mathcal{P}_{1, a^{2}}(\widetilde{H})=\left\{1-a^{2}, a^{3} c\right\}$. 
(2) Let $K$ be the subalgebra of $\widetilde{H}$ generated by the elements a,c. Then $K$ is a Hopf subalgebra of $\widetilde{H}$ which is isomorphic to the Hopf algebra $\mathcal{E}_{\mathcal{A}}$ given in [16. Lemma 3.3] or $\mathcal{K}$ given in [15, Proposition 2.1] as Hopf algebras. In particular, $\widetilde{H} \cong K \otimes \mathbb{k}\left[\mathbb{Z}_{2}\right]$ as Hopf algebras.

(3) Let $\mathcal{A}_{1}$ be the pointed Hopf algebra of dimension 16 defined by $\mathcal{A}_{1}:=$ $\mathbb{k}\left\langle g, h, x \mid g h-h g=h^{2}=g^{4}-1=x^{2}-g^{2}+1=g x+x g=h x-x h=0\right\rangle$ with $\Delta(g)=g \otimes g, \Delta(h)=h \otimes h$ and $\Delta(x)=x \otimes g+1 \otimes x$. Let $\mathcal{A}_{4}^{\prime \prime}$ be the Hopf subalgebra of $\mathcal{A}_{1}$ generated by $g, x$. Then $\mathcal{A}_{4}^{\prime \prime}$ is the unique pointed Hopf algebra of dimension 8 with non-pointed dual [28] and $\mathcal{A}_{1} \cong \mathcal{A}_{4}^{\prime \prime} \otimes \mathbb{k}\left[\mathbb{Z}_{2}\right]$. Moreover, $\mathcal{A}_{4}^{\prime \prime} \cong K^{*}$ by [16, Lemma 3.3] and hence $\widetilde{H}^{*} \cong \mathcal{A}_{4}^{\prime \prime} \otimes \mathbb{k}\left[\mathbb{Z}_{2}\right] \cong \mathcal{A}_{1}$.

(4) The set $\left\{g^{j}, x g^{j}, j \in \mathbb{I}_{0,3}\right\}$ is a linear basis of $\mathcal{A}_{4}^{\prime \prime}$. Let $\left\{\left(a^{i}\right)^{*},\left(a^{i} c\right)^{*}, i \in\right.$ $\left.\mathbb{I}_{0,3}\right\}$ be the basis of $\mathcal{A}_{4}^{\prime \prime}$ dual to $\left\{a^{i}, a^{i} c, i \in \mathbb{I}_{0,3}\right\}$. By [15, Remark 2.4], the Hopf algebra isomorphism $\phi: \mathcal{A}_{4}^{\prime \prime} \rightarrow K^{*}$ is given by

$$
\phi\left(g^{j}\right)=\sum_{i=0}^{3} \xi^{-i j}\left(a^{i}\right)^{*}, \quad \psi\left(x g^{j}\right)=\sqrt{2} \xi \sum_{i=0}^{3} \xi^{-(i+1) j}\left(a^{i} c\right)^{*} .
$$

Lemma 4.3. Let $H$ and $K$ be finite-dimensional Hopf algebras. Suppose that $V$ or $W$ is a simple object in ${ }_{H}^{H} \mathcal{Y} \mathcal{D}$ or ${ }_{K}^{K} \mathcal{Y} \mathcal{D}$, respectively. Then $V \otimes W$ is a simple object in $\begin{array}{r}H \otimes K \\ H \otimes K\end{array}$ object $U \in{ }_{H \otimes K}^{H \otimes K} \mathcal{Y D}, U \cong V \otimes W$ for some simple object $V \in{ }_{H}^{H} \mathcal{Y D}$ and simple object $W \in{ }_{K}^{K} \mathcal{Y} \mathcal{D}$.

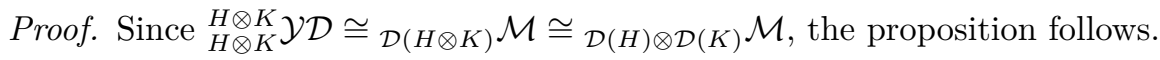

Next, we determine the simple objects in $\underset{\widetilde{H}}{\widetilde{Y}} \mathcal{Y}$. Consider the set $\Omega$ given by

$$
\Omega=\{(i, j, k, \iota) \in \mathbb{N} \times \mathbb{N} \times \mathbb{N} \times \mathbb{N} \mid 0 \leq i, j<4,0 \leq k, \iota<2,2 i \neq j \bmod 4\} .
$$

Clearly, $|\Omega|=48$.

Proposition 4.4. Let $\mathbb{k}_{\lambda_{i, j, k}}=\mathbb{k}\{e\}$ for $(i, j, k) \in \mathbb{I}_{0,1} \times \mathbb{I}_{0,1} \times \mathbb{I}_{0,3}$. Then $\mathbb{k}_{\lambda_{i, j, k}} \in$ $\widetilde{\widetilde{H}} \mathcal{Y} \mathcal{D}$ with the Yetter-Drinfeld module structure given by

$$
a \cdot e=\xi^{k} v, \quad b \cdot e=(-1)^{i}, \quad c \cdot e=0, \quad \delta(e)=a^{2 k} b^{j} \otimes e .
$$

Let $W_{i, j, k, \iota}=\mathbb{k}\left\{e_{1}, e_{2}\right\}$ for $(i, j, k, \iota) \in \Omega$. Then $W_{i, j, k, \iota}$ is a simple object in $\underset{\widetilde{H}}{\widetilde{Y} \mathcal{D}}$ with the Yetter-Drinfeld module structure given by

$$
\begin{aligned}
& a \cdot e_{1}=\xi^{i} e_{1}, \quad b \cdot e_{1}=(-1)^{k} e_{1}, \quad c \cdot e_{1}=0, \\
& a \cdot e_{2}=-\xi^{i+1} e_{2}, \quad b \cdot e_{2}=(-1)^{k} e_{2}, \quad c \cdot e_{2}=e_{1}, \\
& \delta\left(e_{1}\right)=b^{\iota} a^{4-j} \otimes e_{1}+\left(\xi^{3 i}-\xi^{i+j}\right) b^{\iota} a^{1-j} c \otimes e_{2}, \\
& \delta\left(e_{2}\right)=b^{\iota} a^{2-j} \otimes e_{2}+\frac{1}{2}\left(\xi^{i}+\xi^{3 i+j}\right) b^{\iota} a^{3-j} c \otimes e_{1} .
\end{aligned}
$$

Moreover, any simple object $W$ in $\underset{\widetilde{H}}{\widetilde{H}} \mathcal{Y} \mathcal{D}$ is isomorphic to $\mathbb{k}_{\lambda_{i, j, k}}$ for some $(i, j, k) \in$ $\mathbb{I}_{0,1} \times \mathbb{I}_{0,1} \times \mathbb{I}_{0,3}$ or $W_{i, j, k, \iota}$ for some $(i, j, k, \iota) \in \Omega$. 
Proof. It follows by [15, Propositions 3.1 and 3.3] and Lemma 4.3 .

Remark 4.5. By [15, Remark 2.8], $W_{i, j, k, \iota}^{*} \cong W_{-i+1,-j+2, k, \iota}$ for $(i, j, k, \iota) \in \Omega$.

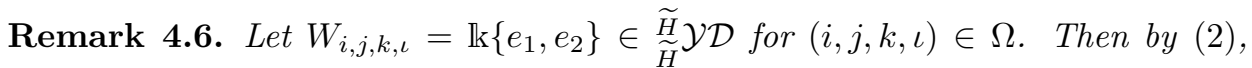
$W_{i, j, k, \iota} \in{ }_{\mathcal{A}_{1}}^{\mathcal{A}_{1}} \mathcal{Y} \mathcal{D}$ with the Yetter-Drinfeld module structure given by

$$
\begin{aligned}
& g \cdot e_{1}=\xi^{-j} e_{1}, \quad h \cdot e_{1}=(-1)^{\iota} e_{1}, \quad x \cdot e_{1}=\sqrt{2} \xi\left(\xi^{-i-j}-\xi^{i}\right) e_{2}, \\
& g \cdot e_{2}=\xi^{2-j} e_{2}, \quad h \cdot e_{2}=(-1)^{\iota} e_{2}, \quad x \cdot e_{2}=-\frac{1}{2}\left(\xi^{i-j}+\xi^{-i}\right) e_{1}, \\
& \delta\left(e_{1}\right)=g^{i} h^{k} \otimes e_{1}, \quad \delta\left(e_{2}\right)=g^{i-1} h^{k} \otimes e_{2}+\frac{\sqrt{2}}{2} \xi^{i-1} x g^{i-1} h^{k} \otimes e_{1} .
\end{aligned}
$$

Now we describe the braidings of the simple objects in $\underset{\tilde{H}}{\widetilde{Y}} \mathcal{D}$.

Proposition 4.7. Let $\mathbb{k}_{\lambda_{i, j, k}}=\mathbb{k}\{e\} \in \underset{\widetilde{H}}{\widetilde{H}} \mathcal{V}$ for $(i, j, k) \in \mathbb{I}_{0,1} \times \mathbb{I}_{0,1} \times \mathbb{I}_{0,3}$. Then the braiding of $\mathbb{k}_{\lambda_{i, j, k}}$ is given by $c(e \otimes e)=(-1)^{i j+k} e \otimes e$.

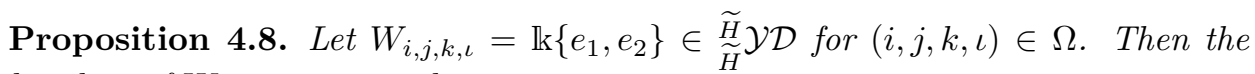
braiding of $W_{i, j, k, \iota}$ is given by

$c\left(\left[\begin{array}{l}e_{1} \\ e_{2}\end{array}\right] \otimes\left[e_{1} e_{2}\right]\right)=(-1)^{k \iota}\left[\begin{array}{cc}\xi^{-i j} e_{1} \otimes e_{1} & \xi^{(1-i) j} e_{2} \otimes e_{1}+s_{12} e_{1} \otimes e_{2} \\ \xi^{i(2-j)} e_{1} \otimes e_{2} & -\xi^{2 i-i j+j} e_{2} \otimes e_{2}+s_{11} e_{1} \otimes e_{1}\end{array}\right]$, where $s_{12}=\xi^{-i j}-\xi^{2 i-i j+j}, s_{11}=\frac{1}{2}\left(\xi^{-i j}+\xi^{2 i-i j+j}\right)$.

Finally, we determine all finite-dimensional Nichols algebras over simple objects in $\underset{H}{\widetilde{H}} \mathcal{Y D}$ and present them by generators and relations. We shall show that all finite-dimensional Nichols algebras over one-dimensional objects in $\underset{H}{\widetilde{H}} \mathcal{Y} \mathcal{D}$ are parametrized by the set

$$
\Omega^{0}=\left\{(i, j, k) \in \mathbb{I}_{0,1} \times \mathbb{I}_{0,1} \times \mathbb{I}_{0,3} \mid 2 \nmid i j+k\right\} .
$$

By Proposition 4.7, the next result follows immediately.

Lemma 4.9. The Nichols algebra $\mathcal{B}\left(\mathbb{k}_{\lambda_{i, j, k}}\right)$ over $\mathbb{k}_{\lambda_{i, j, k}}$ for $(i, j, k) \in \mathbb{I}_{0,1} \times \mathbb{I}_{0,1} \times \mathbb{I}_{0,3}$ is

$$
\mathcal{B}\left(\mathbb{k}_{\lambda_{i, j, k}}\right)= \begin{cases}\mathbb{k}[e] & \text { if }(i, j, k) \in(i, j, k) \in \mathbb{I}_{0,1} \times \mathbb{I}_{0,1} \times \mathbb{I}_{0,3}-\Omega^{0} \\ \bigwedge \mathrm{k}_{\lambda_{i, j, k}} & \text { if }(i, j, k) \in \Omega^{0}\end{cases}
$$

We shall determine all finite-dimensional Nichols algebras over two-dimensional

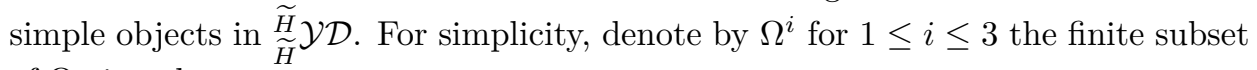
of $\Omega$ given by

$$
\begin{aligned}
& \Omega^{1}=\{(i, j, k, \iota) \in \Omega \mid j=0,2, k=\iota=1\}, \\
& \Omega^{2}=\{(i, j, k, \iota) \in \Omega \mid i-2=k \iota=0 \text { or } i=k-1=\iota-1=0, j \neq 2\}, \\
& \Omega^{3}=\{(i, j, k, \iota) \in \Omega \mid i-3=k \iota=0 \text { or } i-1=k-1=\iota-1=0, j \neq 0\} .
\end{aligned}
$$


It is easy to check that $\left|\Omega^{1}\right|=4,\left|\Omega^{2}\right|=8=\left|\Omega^{3}\right|$ and $\xi^{(1-i) j}(-1)^{k \iota}=-\xi^{j}$ for $(i, j, k, \iota) \in \Omega^{1} \cup \Omega^{2}$. It turns out that the Nichols algebra $\mathcal{B}\left(W_{i, j, k, \iota}\right)$ is finitedimensional for $(i, j, k, \iota) \in \Omega^{1} \cup \Omega^{2} \cup \Omega^{3}$.

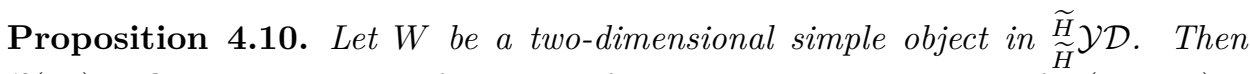
$\mathcal{B}(W)$ is finite-dimensional if and only if $W$ is isomorphic to $W_{i, j, k, \iota}$ for $(i, j, k, \iota) \in$ $\Omega^{1} \cup \Omega^{2} \cup \Omega^{3}$. Moreover, the generators and relations are given by

\begin{tabular}{|c|c|c|}
\hline$(i, j, k, \iota) \in$ & relations of $\mathcal{B}\left(V_{i, j, k, \iota}\right)$ with generators $v_{1}, v_{2}$ & $\operatorname{dim} \mathcal{B}(V)$ \\
\hline$\Omega^{1}$ & $e_{1}^{2}=0, e_{1} e_{2}+\xi^{j} e_{2} e_{1}=0, e_{2}^{2}=0$ & 4 \\
\hline$\Omega^{2}$ & $e_{1}^{2}=0, e_{1} e_{2}+\xi^{j} e_{2} e_{1}=0, e_{2}^{4}=0$ & 8 \\
\hline$\Omega^{3}$ & $e_{1}^{4}=0, e_{1} e_{2}+e_{2} e_{1}=0, e_{1}^{2}-2 e_{2}^{2}=0$ & 8 \\
\hline
\end{tabular}

Proof. Assume that $(i, j, k, \iota) \in \Omega-\cup_{i=1}^{3} \Omega^{i}$; we claim that $\operatorname{dim} \mathcal{B}\left(W_{i, j, k, \iota}\right)=\infty$. Indeed, a direct computation shows that $(-1)^{k \iota} \xi^{-i j}=1$ or $(-1)^{k \iota} \xi^{2 i-i j+j}=-1$. If $(-1)^{k \iota} \xi^{-i j}=1$, then the braiding of $W_{i, j, k, \iota}$ contains an eigenvector $e_{1} \otimes e_{1}$ of eigenvalue 1 and hence the claim follows. If $(-1)^{k \iota} \xi^{2 i-i j+j}=-1$, then by Remark 4.5 and Proposition 2.3 the claim follows.

Assume that $(i, j, k, \iota) \in \cup_{i=1}^{3} \Omega^{i}$. Then the braided vector space $W_{i, j, k, \iota}$ belongs to the case $\mathfrak{R}_{2,1}, \mathfrak{R}_{1,2}$ or $\mathfrak{R}_{1,2}(a)$ in [4] for $(i, j, k, \iota) \in \Omega^{1}, \Omega^{2}$ or $\Omega^{3}$, respectively. More precisely,

- If $(i, j, k, \iota) \in \Omega^{1}$, then $t^{2}=\xi^{i j}(-1)^{k \iota}=-1, t q=\xi^{(1-i) j}(-1)^{k \iota}=-\xi^{j}$, $t p=\xi^{i(2-j)}(-1)^{k \iota}=(-1)^{i+1}$;

- if $(i, j, k, \iota) \in \Omega^{2}$, then $p=-1, q=\xi^{(1-i) j}(-1)^{k \iota}=-\xi^{j}, k=\frac{1}{2}(p+q)$;

- if $(i, j, k, \iota) \in \Omega^{3}$, then $q=(-1)^{k \iota} \xi^{i(2-j)}, p=-1, k=-\frac{1}{2}(q+p)$.

Then by [4, Proposition 3.3, 3.10 and 3.11], the assertion follows.

Remark 4.11. By Remark 4.5 and Proposition 2.3. $\mathcal{B}\left(W_{i, 2,1,1}\right) \cong \mathcal{B}\left(W_{1-i, 0,1,1}\right)^{* \text { bop }}$ and $\mathcal{B}\left(W_{i, j, k, \iota}\right) \cong \mathcal{B}\left(W_{1-i, j, k, \iota}\right)^{* b o p}$, where $j=1,3, i=0,2$ and $k, \iota \in\{0,1\}$. From the proof of Proposition 4.10, the braiding matrices of $W_{i, j, k, \iota}$ for $(i, j, k, \iota) \in \Omega^{2}$ are, up to a primitive 4 th root of unity, the same and so are for $(i, j, k, \iota) \in \Omega^{3}$. The Nichols algebras $\mathcal{B}\left(W_{i, j, k, \iota}\right)$ for $(i, j, k, \iota) \in \Omega^{2} \cup \Omega^{3}$ have already appeared in [15], and $\mathcal{B}\left(W_{i, j, k, \iota}\right)$ with $(i, j, k, \iota) \in \Omega^{1}$ are isomorphic to the Nichols algebras $\mathcal{B}\left(V_{i, j, k, \iota}\right)$ with $(i, j, k, \iota) \in \Lambda^{2}$.

Remark 4.12. It should be pointed out that $\mathcal{B}\left(W_{i, j, k, 0}\right) \sharp K$ is a Hopf subalgebra of $\mathcal{B}\left(W_{i, j, k, 0}\right) \sharp \widetilde{H}$ for $(i, j, k, 0) \in \Omega^{1} \cup \Omega^{2} \cup \Omega^{3}$ and $\mathcal{B}\left(W_{i, j, 0,0}\right) \sharp \widetilde{H} \cong \mathcal{B}\left(W_{i, j, 0,0}\right) \sharp K \otimes$ $\mathbb{k}\left[\mathbb{Z}_{2}\right]$ as Hopf algebras, where $i \in\{2,3\}, j \in\{1,3\}$.

Remark 4.13. Note that gr $\mathcal{A}_{4}^{\prime \prime} \cong\left(\mathcal{A}_{4}^{\prime \prime}\right)_{\sigma}$ for some Hopf 2-cocycle $\sigma$. By [19, Proposition 4.2],

$$
\sigma=\epsilon \otimes \epsilon-\zeta, \quad \text { where } \zeta\left(x^{i} g^{j}, x^{k} g^{l}\right)=(-1)^{j k} \delta_{2, i+k} \text { for } i, k \in \mathbb{I}_{0,1}, j, l \in \mathbb{I}_{0,3} .
$$

Similar to Remark 3.17, the Nichols algebras of dimension greater than 2 in Proposition 4.10 can be related to the Nichols algebras $\mathcal{B}\left(Y_{i, j, k, \iota}\right)$ of diagonal type. More 
precisely, if $(i, j, k, \iota) \in \Omega^{1}$, then the Dynkin diagram of $\mathcal{B}\left(Y_{i, j, k, \iota}\right)$ is $0^{-1}-1$ If $(i, j, k, \iota) \in \Omega^{2}$, then the Dynkin diagram is $\begin{gathered}-1 \\ 0\end{gathered} \stackrel{\xi^{ \pm j}}{-1}$ - . If $(i, j, k, \iota) \in \Omega^{3}$, then

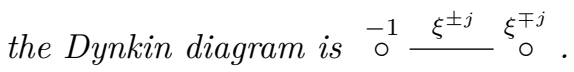

4.2. Finite-dimensional Hopf algebras over $\widetilde{H}$. In this subsection, we determine all finite-dimensional Hopf algebras over $\widetilde{H}$ such that their diagrams are strictly graded and their infinitesimal braidings are simple objects in $\underset{\widetilde{H}}{\widetilde{H}} \mathcal{Y}$. We first show that the diagrams of these Hopf algebras are Nichols algebras.

Lemma 4.14. Let $A$ be a finite-dimensional Hopf algebra over $\widetilde{H}$ such that the

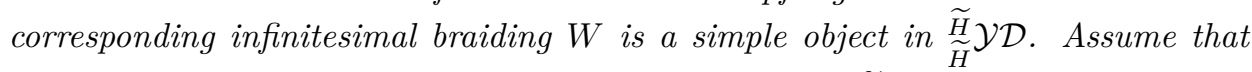
the diagram of $A$ is strictly graded. Then $\operatorname{gr} A \cong \mathcal{B}(W) \sharp \widetilde{H}$.

Proof. Similar to the proof of Proposition 3.18

Next, we shall show that there do not exist non-trivial liftings for the bosonizations of the Nichols algebras over $\mathbb{k}_{\lambda_{i, j, k}}$ with $(i, j, k) \in \Omega^{0}$, and over $W_{i, j, k, \iota}$ with $(i, j, k, \iota) \in \Omega^{1} \cup \Omega^{2}$.

Proposition 4.15. Let $A$ be a finite-dimensional Hopf algebra over $\widetilde{H}$ such that $\operatorname{gr} A \cong \mathcal{B}(W) \sharp \widetilde{H}$, where $W$ is isomorphic either to $\mathbb{k}_{\lambda_{i, j, k}}$ for $(i, j, k) \in \Omega^{0}$ or to $W_{i, j, k, \iota}$ for $(i, j, k, \iota) \in \Omega^{1} \cup \Omega^{2}$. Then $A \cong \operatorname{gr} A$.

Proof. We prove the assertion by showing that the defining relations of gr $A$ hold in $A$. Assume that $W \cong W_{i, j, k, \iota}$ for $(i, j, k, \iota) \in \Omega^{1}$. Note that $\mathcal{B}\left(W_{i, j, k, \iota}\right) \sharp \widetilde{H}$ is generated by $x, y, a, b, c$ satisfying the relations $(9)$ and the relations

$$
\begin{gathered}
a x=\xi^{i} x a, \quad b x=(-1)^{k} x b, \quad c x=\xi^{3 i} x c, \quad a y+\xi^{i+1} y a=(-1)^{i} x c, \\
b y=(-1)^{k} y b, \quad c y+\xi^{3(i+1)} y c=x a, \quad x^{2}=0, \quad x y+\xi^{j} y x=0, \quad y^{2}=0,
\end{gathered}
$$

with the coalgebra structure given by 10$), \Delta(x)=x \otimes 1+b^{\iota} a^{4-j} \otimes x+\left(\xi^{3 i}-\right.$ $\left.\xi^{i+j}\right) b^{\iota} a^{1-j} c \otimes y$ and $\Delta(y)=y \otimes 1+b^{\iota} a^{2-j} \otimes y+\frac{1}{2}\left(\xi^{i}+\xi^{3 i+j}\right) b^{\iota} a^{3-j} c \otimes x$.

A direct computation shows that $y^{2} \in \mathcal{P}(A)$ and $x y+\xi^{j} y x \in \mathcal{P}_{1, a^{2}}(A)$. Then $y^{2}=0$ in $A$. Since $\mathcal{P}_{1, a^{2}}(A)=\mathcal{P}_{1, a^{2}}\left(\mathcal{B}\left(V_{i, j, k, \iota}\right) \sharp \widetilde{H}\right)=\mathcal{P}_{1, a^{2}}(\widetilde{H})=\mathbb{k}\left\{1-a^{2}, a^{3} c\right\}$, it follows that $x y+\xi^{j} y x=\mu\left(1-a^{2}\right)+\nu a^{3} c$ for some $\mu, \nu \in \mathbb{k}$. Since $a\left(x y+\xi^{j} y x\right)=$ $\xi^{2 i+1}\left(x y+\xi^{j} y x\right) a$ and $c\left(x y+\xi^{j} y x\right)=\xi^{2 i-1}\left(x y+\xi^{j} y x\right) c$, it follows that $\mu=\nu=0$ and hence $x y+\xi^{j} y x=0$ in $A$. Finally, $\Delta\left(x^{2}\right)=x^{2} \otimes 1+1 \otimes x^{2}$, which implies that the relation $x^{2}=0$ holds in $A$. Consequently, the assertion follows.

For $W \cong W_{i, j, k, \iota}$ for $(i, j, k, \iota) \in \Omega^{2}$ or $\mathbb{k}_{\lambda_{i, j, k}}$ for $(i, j, k) \in \Omega^{0}$, the proof follows the same lines as $W_{i, j, k, \iota}$ for $(i, j, k, \iota) \in \Omega^{1}$.

Now we define eight families of Hopf algebras $\Omega_{i, j, k, \iota}^{3}(\mu)$ with $(i, j, k, \iota) \in \Omega^{3}$ and show that they are indeed liftings of the Nichols algebras $\mathcal{B}\left(W_{i, j, k, \iota}\right)$ with $(i, j, k, \iota) \in \Omega^{3}$. 
Definition 4.16. For $\mu \in \mathbb{k}$ and $(i, j, k, \iota) \in \Omega^{3}$, let $\Omega_{i, j, k, \iota}^{3}(\mu)$ be the algebra generated by $x, y, a, b, c$, satisfying the relations (9) and the following ones:

$a x=\xi^{i} x a, \quad b x=(-1)^{k} x b, \quad c x=\xi^{3 i} x c, \quad a y+\xi^{i+1} y a=(-1)^{i} x c, \quad b y=(-1)^{k} y b$,

$c y+\xi^{3(i+1)} y c=x a, \quad x^{4}=0, \quad x y+y x=-\mu \xi^{i} a c, \quad x^{2}-2 y^{2}=\mu\left(1-a^{2}\right)$.

$\Omega_{i, j, k, \iota}^{3}(\mu)$ is a Hopf algebra with the coalgebra structure given by 10 and

$$
\begin{aligned}
& \Delta(x)=x \otimes 1+b^{\iota} a^{4-j} \otimes x+\left(\xi^{3 i}-\xi^{i+j}\right) b^{\iota} a^{1-j} c \otimes y, \\
& \Delta(y)=y \otimes 1+b^{\iota} a^{2-j} \otimes y+\frac{1}{2}\left(\xi^{i}+\xi^{3 i+j}\right) b^{\iota} a^{3-j} c \otimes x .
\end{aligned}
$$

Remark 4.17. (1) It is clear that $\Omega_{i, j, k, \iota}^{3}(0) \cong \mathcal{B}\left(W_{i, j, k, \iota}\right) \sharp \widetilde{H}$ and $\Omega_{i, j, k, \iota}^{3}(\mu)$ with $\mu \neq 0$ is not isomorphic to $\Omega_{i, j, k, \iota}^{3}(0)$ for $(i, j, k, \iota) \in \Omega^{3}$.

(2) Denote by $\overline{\Omega_{i, j, k, \iota}^{3}(\mu)}$ the subalgebra of $\Omega_{i, j, k, \iota}^{3}(\mu)$ generated by $a, c, x$ and $y$. It is clear that $\Omega_{i, j, 0, \iota}^{3}(\mu) \cong \overline{\Omega_{i, j, 0, \iota}^{3}(\mu)} \otimes \mathbb{k}\left[\mathbb{Z}_{2}\right]$ as algebras but not as coalgebras. Moreover, $\Omega_{i, j, 0,0}^{3}(\mu) \cong \overline{\Omega_{i, j, 0,0}^{3}(\mu)} \otimes \mathbb{k}\left[\mathbb{Z}_{2}\right]$ as Hopf algebras. In particular, $\overline{\Omega_{i, j, 0,0}^{3}(\mu)}$ is isomorphic to the Hopf algebra $\mathfrak{A}_{i, j}(\mu)$ in [15, Definitions 5.4/5.6].

Lemma 4.18. A linear basis of $\Omega_{i, j, k, \iota}^{3}(\mu)$ is given by

$$
\left\{y^{r} x^{s} a^{t} b^{u} c^{v}, s, t \in \mathbb{I}_{0,3}, r, u, v \in \mathbb{I}_{0,1}\right\} .
$$

In particular, $\operatorname{dim} \Omega_{i, j, k, \iota}^{3}(\mu)=128$.

Proof. By the Diamond Lemma, it suffices to show that all overlaps ambiguities are resolvable with the order $y<x<a<b<c<d$. Here we only show that $(x y) y=x\left(y^{2}\right),\left(x^{4}\right) y=x^{3}(x y)$ are resolvable and the others are completely similar.

After a direct computation, we have that $(a c) y-y(a c)=\xi^{i} x a^{2}$. Then

$$
\begin{aligned}
(x y) y & =\left(-y x-\mu \xi^{i} a c\right) y=-y x y-\mu \xi^{i} a c y=-y\left(-y x-\mu \xi^{i} a c\right)-\mu \xi^{i} a c y \\
& =y^{2} x+\mu \xi^{i}(y a c-a c y)=y^{2} x-\mu \xi^{2 i} x a^{2}=y^{2} x+\mu x a^{2} \\
& =\frac{1}{2}\left[x^{2}-\mu\left(1-a^{2}\right)\right] x+\mu x a^{2}=\frac{1}{2} x^{3}-\frac{1}{2} \mu\left(1-a^{2}\right) x+\mu x a^{2} \\
& =\frac{1}{2} x^{3}-\frac{1}{2} \mu x+\frac{1}{2} \mu x a^{2}=x\left(y^{2}\right) .
\end{aligned}
$$

Note that $a c x=x a c$, then

$x^{2} y=-x y x-\mu \xi^{i} x a c=\left(y x+\mu \xi^{i} a c\right) x-\mu \xi^{i} x a c=y x^{2}+\mu \xi^{i} a c x-\mu \xi^{i} x a c=y x^{2}$.

Therefore $x^{3}(x y)=y x^{4}=0=y\left(x^{4}\right)$. It follows that the overlaps $(x y) y=$ $x\left(y^{2}\right),\left(x^{4}\right) y=x^{3}(x y)$ are resolvable.

Now we show that $\Omega_{i, j, k, \iota}^{3}(\mu)$ is a lifting of the bosonization $\mathcal{B}\left(W_{i, j, k, \iota}\right) \sharp \widetilde{H}$ for $(i, j, k, \iota) \in \Omega^{3}$.

Lemma 4.19. For $(i, j, k, \iota) \in \Omega^{3}$, gr $\Omega_{i, j, k, \iota}^{3}(\mu) \cong \mathcal{B}\left(W_{i, j, k, \iota}\right) \sharp \widetilde{H}$. 
Proof. Similar to the proof of Proposition 3.25

Proposition 4.20. Let $A$ be a finite-dimensional Hopf algebra over $\widetilde{H}$ such that $\operatorname{gr} A \cong \mathcal{B}(W) \sharp \widetilde{H}$, where $W$ is isomorphic to $W_{i, j, k, \iota}$ for $(i, j, k, \iota) \in \Omega^{3}$. Then $A \cong \Omega_{i, j, k, L}^{3}(\mu)$.

Proof. Note that gr $A \cong \Omega_{i, j, k, \iota}^{3}(0)$ as Hopf algebras. As $\Delta(x)=x \otimes 1+b^{\iota} a^{4-j} \otimes$ $x+\left(\xi^{3 i}-\xi^{i+j}\right) b^{\iota} a^{1-j} c \otimes y$ and $\Delta(y)=y \otimes 1+b^{\iota} a^{2-j} \otimes y+\frac{1}{2}\left(\xi^{i}+\xi^{3 i+j}\right) b^{\iota} a^{3-j} c \otimes x, \mathrm{a}$ direct computation shows that $x^{2}-2 y^{2} \in \mathcal{P}_{1, a^{2}}(A)=\mathcal{P}_{1, a^{2}}(\widetilde{H})$. Since $\mathcal{P}_{1, a^{2}}(\widetilde{H})=$ $\mathbb{k}\left\{1-a^{2}, a^{3} c\right\}$, we have $x^{2}-2 y^{2}=\mu\left(1-a^{2}\right)+\nu a^{3} c$ for some $\mu, \nu \in \mathbb{k}$. Furthermore, it follows by a direct computation that

$\Delta\left(x y+y x+\xi^{i} \mu a c\right)=\left(x y+y x+\xi^{i} \mu a c\right) \otimes 1+1 \otimes\left(x y+y x+\xi^{i} \mu a c\right)+\nu a c \otimes a^{3} c$.

Then a tedious computation on $A_{[1]}$ shows that the last equation holds only if $\nu=0$, which implies that $x y+y x+\xi^{i} \mu a c=0$ and $x^{2}-2 y^{2}=\mu\left(1-a^{2}\right)$ in $A$. Finally, $\Delta\left(x^{4}\right)=\Delta(x)^{4}=x^{4} \otimes 1+1 \otimes x^{4}$ and hence $x^{4}=0$ in $A$.

Since the defining relations of $\Omega_{i, j, k, L}^{3}(\mu)$ hold in $A$, there is a Hopf algebra epimorphism from $\Omega_{i, j, k, \iota}^{3}(\mu)$ to $A$. By Lemma $4.18 \operatorname{dim} A=\operatorname{dim} \Omega_{i, j, k, \iota}^{3}(\mu)$ and hence $A \cong \Omega_{i, j, k, \iota}^{3}(\mu)$.

Finally, we have the classification of finite-dimensional Hopf algebras over $\widetilde{H}$ such that their diagrams are strictly graded and their infinitesimal braidings are simple objects in $\underset{\widetilde{H}}{\widetilde{H}} \mathcal{Y}$.

Proof of Theorem B. The Hopf algebras from different families are pairwise non-isomorphic since their infinitesimal braidings are pairwise non-isomorphic as Yetter-Drinfeld modules over $\widetilde{H}$. And the rest of assertions follow by Lemmas 4.9 and 4.14, and Propositions 4.10, 4.15 and 4.20

\section{ACKNOWLEDGMENT}

The authors are grateful to N. Andruskiewitsch and G. A. García for their kind comments. The authors would like to thank the referee for the helpful comments and suggestions that largely improved the exposition.

\section{REFERENCES}

[1] N. Andruskiewitsch, I. Angiono, On Nichols algebras over basic Hopf algebras, preprint, 2018. arXiv:1802.00316 [math.QA].

[2] N. Andruskiewitsch and M. Beattie, Irreducible representations of liftings of quantum planes. In: Lie theory and its applications in physics V, 414-423, World Sci. Publ., River Edge, NJ, 2004. MR 2172918.

[3] N. Andruskiewitsch and J. Cuadra, On the structure of (co-Frobenius) Hopf algebras, J. Noncommut. Geom. 7 (2013), no. 1, 83-104. MR 3032811

[4] N. Andruskiewitsch and J. M. J. Giraldi, Nichols algebras that are quantum planes, Linear and Multilinear Algebra 66 (2018), no. 5, 961-991. MR 3775317 
[5] N. Andruskiewitsch and M. Graña, Braided Hopf algebras over non-abelian finite groups, Bol. Acad. Nac. Cienc. (Córdoba) 63 (1999), 45-78. MR 1714540

[6] N. Andruskiewitsch, I. Heckenberger and H.-J. Schneider, The Nichols algebra of a semisimple Yetter-Drinfeld module, Amer. J. Math. 132 (2010), no. 6, 1493-1547. MR 2766176

[7] N. Andruskiewitsch and H.-J. Schneider, Lifting of quantum linear spaces and pointed Hopf algebras of order $p^{3}$, J. Algebra 209 (1998), no. 2, 658-691. MR 1659895

[8] — Pointed Hopf algebras. In: New directions in Hopf algebras, 1-68, Math. Sci. Res. Inst. Publ., 43, Cambridge Univ. Press, Cambridge, 2002. MR 1913436

[9] I. Angiono, On Nichols algebras of diagonal type, J. Reine Angew. Math. 683 (2013), 189-251. MR 3181554

[10] - A presentation by generators and relations of Nichols algebras of diagonal type and convex orders on root systems, J. Eur. Math. Soc. 17 (2015), no. 10, 2643-2671. MR 3420518

[11] M. Beattie, Duals of pointed Hopf algebras, J. Algebra 262 (2003), no. 1, 54-76. MR 1970802

[12] M. Beattie and G. A. García, Classifying Hopf algebras of a given dimension. In: Hopf algebras and tensor categories, 125-152, Contemp. Math., 585, Amer. Math. Soc., Providence, RI, 2013. MR 3077235

[13] G. Bergman, The diamond lemma for ring theory, Adv. Math. 29 (1978), no. 2, 178-218. MR 0506890

[14] S. Caenepeel, S. Dăscălescu and Ş. Raianu, Classifying pointed Hopf algebras of dimension 16, Comm. Algebra 28 (2000), no. 2, 541-568. MR 1736746

[15] G. A. García and J. M. J. Giraldi, On Hopf algebras over quantum subgroups, J. Pure Appl. Algebra, to appear. Online: https://doi.org/10.1016/j.jpaa.2018.04.018

[16] G. A. García and C. Vay, Hopf algebras of dimension 16, Algebr. Represent. Theory 13 (2010), no. 4, 383-405. MR 2660853

[17] M. Graña, A freeness theorem for Nichols algebras, J. Algebra 231 (2000), no. 1, 235-257. MR 1779599

[18] L. Grunenfelder and M. Mastnak, Pointed and copointed Hopf algebras as cocycle deformations, 2007. arXiv:0709.0120 [math.RA].

[19] - Pointed Hopf algebras as cocycle deformations, 2010. arXiv:1010.4976 [math.QA].

[20] I. Heckenberger, Classification of arithmetic root systems, Adv. Math. 220 (2009), no. 1, 59-124. MR 2462836

[21] I. Heckenberger and H.-J. Schneider, Yetter-Drinfeld modules over bosonizations of dually paired Hopf algebras, Adv. Math. 244 (2013), 354-394. MR 3077876

[22] J. Hietarinta, Solving the two-dimensional constant quantum Yang-Baxter equation, J. Math. Phys. 34 (1993), no. 5, 1725-1756. MR 1214484

[23] N. Hu and R. Xiong, Eight classes of new Hopf algebras of dimension 128 without the Chevalley property, 2017. arXiv:1701.01991 [math.QA].

[24] S. Majid and R. Oeckl, Twisting of quantum differentials and the Planck scale Hopf algebra, Comm. Math. Phys. 205 (1999), no. 3, 617-655. MR 1711340

[25] S. Montgomery, Hopf algebras and their actions on rings, CBMS Regional Conference Series in Mathematics, 82, American Mathematical Society, Providence, RI, 1993. MR 1243637.

[26] D. E. Radford, The structure of Hopf algebras with a projection, J. Algebra 92 (1985), no. 2, 322-347. MR 0778452

[27] - Hopf algebras, Series on Knots and Everything, 49, World Scientific, Hackensack, NJ, 2012. MR 2894855 
[28] D. Ştefan, Hopf algebras of low dimension, J. Algebra 211 (1999), no. 1, 343-361. MR 1656583

[29] S. Ufer, Triangular braidings and pointed Hopf algebras, J. Pure Applied Algebra 210 (2007), no. 2, 307-320. MR 2320000

N. $H u$

School of Mathematical Sciences, Shanghai Key Laboratory of PMMP, East China Normal University, Shanghai 200241, China

nhhu@math.ecnu.edu.cn

R. Xiong $\bowtie$

School of Mathematical Sciences, Shanghai Key Laboratory of PMMP, East China Normal University, Shanghai 200241, China

rcxiong@foxmail.com

Received: May 25, 2017

Accepted: June 26, 2018 